\title{
An a priori error estimate for a temporally discontinuous Galerkin space-time finite element method for linear elasto- and visco-dynamics*
}

\author{
Simon Shaw ${ }^{\dagger}$
}

March 19, 2019

${ }^{*}$ The work of Shaw was supported in part by the Engineering and Physical Sciences research council EP/H011072/1. This support is gratefully acknowledged.

${ }^{\dagger}$ simon.shaw@brunel.ac.uk, BICOM, Brunel University London, Uxbridge, UB8 3PH, England. ww. brunel.ac.uk/bicom 


\begin{abstract}
We extend the formulation and a priori error analysis given by Johnson (Discontinuous Galerkin finite element methods for second order hyperbolic problems, Comp. Meth. Appl. Mech. Eng., 107:117-129, 1993) from the acoustic wave equation to a Voigt and Maxwell-Zener viscodynamic system incorporating Rayleigh damping. The elastic term in the Rayleigh damping introduces a multiplicative $T^{1 / 2}$ growth in the constant but otherwise the error bound is consistent with that obtained by Johnson, with a constant that grows a priori with $T^{1 / 2}$ and also with norms of the solution. Gronwall's inequality is not used and so we can expect that this bound is of high enough quality to afford confidence in longtime integration. The viscoelasticity is modelled by internal variables that evolve according to ordinary differential equations and so the system shares similarities with dispersive Debye and Drude metamaterial models currently being studied in electromagnetism, as well as to acoustic metamaterial systems. This appears to be the first time an a priori error analysis has been given for DG-in-time treatment of dispersive problems of this type.
\end{abstract}

Keywords: discontinuous Galerkin, finite element method, a priori error estimate, duality, viscoelasticity, dispersion.

Sub. class: $65 \mathrm{M} 60,45 \mathrm{~K} 05,45 \mathrm{D} 05,74 \mathrm{D} 05,35 \mathrm{Q} 74$

\title{
Contents
}

1 Introduction 3

2 The continuum problem 4

3 The discrete scheme $\quad 8$

4 A priori error estimates 10

$\begin{array}{lll}5 & \text { Implementation and results } & 19\end{array}$

6 Conclusions 22 


\section{Introduction}

In [12] Johnson formulated a space-time finite element method for the acoustic wave equation using a continuous Galerkin (CG) discretization in space and a discontinuous Galerkin (DG) discretization in time - DGCG-FEM. Both a priori and a posteriori error estimates were derived using approximation-error estimates, error representation through a discrete or continuous dual problem, and the associated strong stability of the dual solutions.

Here we extend that formulation to the equations of linear elastodynamics with generic Rayleigh damping, and also with viscoelastic damping provided by either or both of a Voigt term and a Maxwell-Zener history integral with a (Prony series) kernel of decaying exponentials. This Volterra integral is not itself included in the model but is instead captured by internal variables that evolve according to a set of ordinary differential equations.

We note that the Prony series model of viscoelasticity allows for an efficient numerical scheme in so much as we can compute over $N$ time levels using $O(N)$ operations. On the other hand, alternative viscoelastic kernels based on the fractional calculus, or power laws as in [5], require a quadrature summation over time levels $0,1, \ldots, n$ for each time level $n=1, \ldots, N$ and, if implemented naïvely, will require $O\left(N^{2}\right)$ operations. This and the associated computer memory requirements imply that long-time computations in, say, 3D over moderate to long time scales are impractical without using a method that mitigates this difficulty. For example the sparse method in [26] or the convolution quadrature in [24], are available for finite difference time discretizations, and in [20] McLean has proposed a fast method that is economical on storage for a DGFEM time discretization of a subdiffusion equation. These methods are of great interest because, in particular, the Prony series kernels used in viscoelastic models are sometimes felt to decay too fast to be effective in modelling 'real' materials, and may not display the correct frequency dependence (see e.g. [5]).

Nevertheless, the model described, analyzed and implemented below is of considerable importance in modelling damping and frequency dependence in dispersive 'soft' media, e.g. [10, 11], and has very close analogies in dispersive (e.g. Debye, Drude or Lorentz) electromagnetic metamaterial models, e.g. [16, 6, 15, 25]. Moreover, the emergence of negative dynamic mass metamaterials, e.g [29], will also involve the elastodynamic equations with the 'meta-effects' provided by companion evolution equations for, in essence, internal variables. We intend that the extension of the material in [12] offered here will provide a template for the subsequent DGCG-FEM computer modelling and numerical analysis of dispersive media as modelled by internal variable systems.

This extension is not completely trivial which is why we present it here. Some care has to be taken in how the internal variables are defined, see Remark 2.2, because this impacts on the ease with which stability estimates for the dual problem can be derived. It also affects the nature of the dual problem itself and while we do not claim that the approach below is the only one that can be taken, it seems clear that it is quite amenable to analysis and implementation. However, because this is an extension of [12] we have focussed more on giving details for the new terms that arise in the proofs rather than re-iterate the results in that existing work. 
The plan of the paper is as follows. We outline the physical model and its main features in Section 2, and then give the DGCG-FEM approximation in Section 3. We derive an a priori error bound in Section 4 by following a duality argument and using a strong stability estimate for a discrete dual problem, (30). This stability estimate, Theorem 4.4, does not require Gronwall's lemma and this in turn means that the constant in the error bound does not grow exponentially in time, but only a priori as $T^{1 / 2}$ as found in [12], along with the growth stemming from norms of the exact solution. There is also an additional multiplicative temporal growth of $T^{1 / 2}$ of the constant that is tied to the elastic term in the Rayleigh damping — but this growth does not appear in the error estimate in Theorem 4.5 if this type of damping is not present. In either case, the absence of an $e^{c T}$ growth means that we can expect that this bound is of high enough quality to afford confidence in long-time integration. We give some numerical results in Section 5 and finish with a discussion in Section 6.

The 1993 work by Johnson in [12] appears to have been motivated by Hughes and Hulbert's work [7, 9, 8] in elastodynamics. At around the same time French in [3] gave an alternative approach for a DG-in-time method, and French and Peterson [4] formulated a continuous-in-time approximation. Both of these were for the wave equation as a model problem. Later, Li and Wiberg in [17] gave some numerical demonstrations of how effective Johnson's scheme is for elastodynamics and those comments prompted this study. Furthermore, although we restrict attention to approximations that are piecewise linear in space and time, higher order approximations can be implemented using the decoupling approach described in [28]. A disadvantage of this is that it leads naturally to the challenge of solving complex symmetric systems, as in [14, 13], but Richter, Springer and Vexler in [22] have recently outlined an iterative approach that avoids complex arithmetic.

\section{The continuum problem}

To describe the problem and the constitutive relationship, let the spatial domain $\Omega$ be a time-independent open bounded polytope in $\mathbb{R}^{d}$ for $d=1,2$ or 3 , and let it represent the interior of a homogeneous and isotropic linear viscoelastic compressible body with constant mass density $\varrho$. The boundary, $\partial \Omega$, is partitioned into $\left\{\Gamma_{D}, \Gamma_{N}\right\}$ (also time independent) with Dirichlet boundary values given on the closed set $\Gamma_{D}$ and Neumann boundary values specified on the open (and possibly empty) set $\Gamma_{N}$. As usual we require that $\Gamma_{D} \cap \Gamma_{N}=\varnothing$ and $\Gamma_{D} \cup \Gamma_{N}=\partial \Omega$ and we insist that meas $\partial \Omega\left(\Gamma_{D}\right)>0$. The unit outward normal vector to $\Gamma_{N}$ will be written as $\hat{\boldsymbol{n}}$. To describe the time dependence we set $I:=(0, T]$ and will usually use overdots, as in $\dot{v}$, or subscripts, as in $v_{t}$, to denote partial time differentiation.

The viscoelastic body is acted upon by a system of body forces, $\boldsymbol{f}:=\left(f_{i}(\boldsymbol{x}, t)\right)_{i=1}^{d}$ for $\boldsymbol{x}:=\left(x_{i}\right)_{i=1}^{d} \in \Omega$ and $t \in I$, and a system of surface tractions, $\boldsymbol{g}:=\left(g_{i}(\boldsymbol{x}, t)\right)_{i=1}^{d}$ for $\boldsymbol{x} \in$ $\Gamma_{N}$ and $t \in I$, and we seek the displacement from equilibrium, $\boldsymbol{u}=\left(u_{i}(\boldsymbol{x}, t)\right)_{i=1}^{d}: \Omega \times$ $I \rightarrow \mathbb{R}^{d}$ that results from these forces.

To describe the constitutive relationship we follow the standard literature (e.g. [5, 2]), assume that $t=0$ is a reference time such that $\boldsymbol{u}=\mathbf{0}$ for all $t<0$, and introduce the 
(symmetric) strain tensor,

$$
\varepsilon_{i j}(\boldsymbol{u}):=\frac{1}{2}\left(\frac{\partial u_{i}}{\partial x_{j}}+\frac{\partial u_{j}}{\partial x_{i}}\right)
$$

where in this and below we will usually suppress the explicit display of the $\boldsymbol{x}$ dependence. The (symmetric) stress tensor, $\underline{\boldsymbol{\sigma}}:=\left(\sigma_{i j}\right)_{i, j=1}^{d}$, is then given (e.g. [5]) by either of the following linear functionals of displacement,

$$
\begin{aligned}
\sigma_{i j}(\boldsymbol{u} ; t) & =C_{i j k l} \varepsilon_{k l}(\dot{\boldsymbol{u}}(t))+D_{i j k l}(0) \varepsilon_{k l}(\boldsymbol{u}(t))-\int_{0}^{t} \frac{\partial D_{i j k l}(t-s)}{\partial s} \varepsilon_{k l}(\boldsymbol{u}(s)) d s, \\
& =C_{i j k l} \varepsilon_{k l}(\dot{\boldsymbol{u}}(t))+D_{i j k l}(t) \varepsilon_{k l}(\boldsymbol{u}(0))+\int_{0}^{t} D_{i j k l}(t-s) \varepsilon_{k l}(\dot{\boldsymbol{u}}(s)) d s
\end{aligned}
$$

where an integration by parts shows these to be formally equivalent. Here and below summation is implied over repeated indices.

In this $\underline{\boldsymbol{C}}$ and $\underline{\boldsymbol{D}}(t)$ are fourth order tensors with the former related to Kelvin-Voigt viscoelasticity and the latter to the Zener and Maxwell models. In fact $\underline{\boldsymbol{D}}$ is essentially a stress relaxation analogue of the Hooke tensor from linear elasticity and, with $\underline{\boldsymbol{C}}=\underline{\mathbf{0}}$, this is linear elasticity with memory.

In general we assume that $\underline{\boldsymbol{D}}(0)$ is positive definite so that $\gamma_{i j} \gamma_{k l} D_{i j k l}(0)>0$ a.e. in $\Omega$ for all non-zero symmetric second order tensors $\underline{\gamma}$ and also that (on physical grounds) $\underline{\boldsymbol{D}}$ satisfies the symmetries: $D_{i j k l}(t)=D_{j i k l}(t)=D_{i j l k}(t)$. In general $D_{i j k l}(t) \neq D_{k l i j}(t)$ except at $t=0$ and at the limit $t \rightarrow \infty$, but for isotropic materials this last symmetry holds for all times (see e.g. [18, equations (1.10), (2.62)]).

A much simpler formulation entails if we assume that the material is synchronous. This means that every component of $\underline{\boldsymbol{D}}$ has the same time dependence and means that we can replace $\underline{\boldsymbol{D}}(t)$ with the factorization $\varphi(t) \underline{\boldsymbol{D}}$. Now $\underline{\boldsymbol{D}}$ is temporally constant and $\varphi$ is a stress relaxation function which in the material below we take as given by the Prony series

$$
\varphi(t)=\varphi_{0}+\sum_{q=1}^{N_{\varphi}} \varphi_{q} \exp \left(-t / \tau_{q}\right)
$$

where $\varphi_{q} \geqslant 0$ for $q \in\left\{0,1, \ldots, N_{\varphi}\right\}, \tau_{q}>0$ for $q \in\left\{1, \ldots, N_{\varphi}\right\}$ and we normalize so that $\varphi_{0}+\sum_{q} \varphi_{q}=1$. In [5], Golden and Graham observe that $\varphi_{0}=0$ corresponds to a (very slow moving) viscoelastic fluid whereas $\varphi_{0}>0$ gives a solid. We restrict ourselves to synchronous solids below.

Moreover, due to the body being homogeneous and isotropic the tensor $\underline{\boldsymbol{D}}$ can be described by just two Lamé coefficients, $\lambda=\nu E /((1+\nu)(1-2 \nu))$ and $\mu=2 G=$ $E /(1+\nu)$, where $E>0$ is Young's modulus, $G>0$ is the shear modulus and $\nu \in$ $(-1,1 / 2)$ is Poisson's ratio. The case $\nu<0$ allows for auxetic meta-materials, but we can expect that $\nu>0$ for most (if not all) naturally occurring materials. The action of $\underline{\boldsymbol{D}}$ is now given by $D_{i j k l} \varepsilon_{k l}(\boldsymbol{u})=\lambda \nabla \cdot \boldsymbol{u} \delta_{i j}+\mu \varepsilon_{i j}(\boldsymbol{u})$. We assume for simplicity that $\lambda$ and $\mu$ are constant in space and time.

The form of $\underline{\boldsymbol{C}}$ is not so clear cut but in Rayleigh damping (see e.g. Li and Wiberg [17]) we add a term proportional to $\underline{\boldsymbol{\varepsilon}}(\dot{\boldsymbol{u}})$ (a 'stiffness matrix' term) and a term proportional to $\dot{\boldsymbol{u}}$ (a 'mass matrix' term) to the momentum balance. To incorporate the stiffness part 
of this into our model we choose $\underline{\boldsymbol{C}}=\gamma_{E} \underline{\boldsymbol{D}}$ where $\gamma_{E}$ (in units of sec) is a non-negative constant.

Introducing initial data $\breve{\boldsymbol{u}}$ and $\breve{\boldsymbol{w}}$, the resulting problem is, for each $i \in\{1, \ldots, d\}$, find $\boldsymbol{u}$ such that,

$$
\begin{gathered}
\varrho \dot{w}_{i}+\varrho \gamma_{M} w_{i}-\sigma_{i j, j}=f_{i} \quad \text { in } \Omega \times I, \\
\boldsymbol{w}=\dot{\boldsymbol{u}}, \quad \boldsymbol{u}(0)=\breve{\boldsymbol{u}}, \quad \boldsymbol{w}(0)=\breve{\boldsymbol{w}} \\
\boldsymbol{u}=\mathbf{0} \text { on } \Gamma_{D} \times I \quad \text { and } \quad \sigma_{i j} \widehat{n}_{j}=g_{i} \text { on } \Gamma_{N} \times I
\end{gathered}
$$

where the $\gamma_{M}$ term is the 'mass matrix' contribution to the Rayleigh damping, for $\gamma_{M}$ (in units of $\mathrm{sec}^{-1}$ ) a non-negative constant.

We could work with the memory integrals, (2) or (3), for the constitutive time dependence, but when the stress relaxation function is given by (44) we can capture the history with internal variables. For this we set $\beta_{q}:=\left(\varphi_{q} \tau_{q}\right)^{1 / 2}$ and note that

$$
\varphi(t) \underline{\varepsilon}(\breve{\boldsymbol{u}})+\int_{0}^{t} \varphi(t-s) \underline{\boldsymbol{\varepsilon}}(\dot{\boldsymbol{u}}(s)) d s=\left(\varphi(t)-\varphi_{0}\right) \underline{\boldsymbol{\varepsilon}}(\breve{\boldsymbol{u}})+\varphi_{0} \underline{\boldsymbol{\varepsilon}}(\boldsymbol{u}(t))+\sum_{q=1}^{N_{\varphi}} \beta_{q} \underline{\varepsilon}\left(\boldsymbol{z}_{q}(t)\right),
$$

where the internal variables are defined as,

$$
\boldsymbol{z}_{q}(t):=\int_{0}^{t}\left(\frac{\varphi_{q}}{\tau_{q}}\right)^{1 / 2} \dot{\boldsymbol{u}}(s) e^{-(t-s) / \tau_{q}} d s
$$

or, equivalently, recalling that $\boldsymbol{w}:=\dot{\boldsymbol{u}}$,

$$
\boldsymbol{z}_{q}(t)+\tau_{q} \dot{\boldsymbol{z}}_{q}(t)=\beta_{q} \boldsymbol{w}(t), \quad \text { with } \boldsymbol{z}_{q}(0)=\mathbf{0}
$$

for $q=1,2, \ldots, N_{\varphi}$. With this the constitutive law (3) can be written as,

$$
\underline{\boldsymbol{\sigma}}(\boldsymbol{u} ; t)=\gamma_{E} \underline{\boldsymbol{D}} \underline{\boldsymbol{\varepsilon}}(\boldsymbol{w}(t))+\underline{\boldsymbol{D}}\left(\left(\varphi(t)-\varphi_{0}\right) \underline{\boldsymbol{\varepsilon}}(\breve{\boldsymbol{u}})+\varphi_{0} \underline{\boldsymbol{\varepsilon}}(\boldsymbol{u}(t))+\sum_{q=1}^{N_{\varphi}} \beta_{q} \underline{\varepsilon}\left(\boldsymbol{z}_{q}(t)\right)\right) .
$$

To give a weak formulation of (15) with (10) we first recall the product Hilbert spaces, $\boldsymbol{H}^{s}(\Omega):=H^{s}(\Omega)^{d}$, for $s=0,1,2, \ldots$, with inner products given for all $\boldsymbol{w}, \boldsymbol{v} \in \boldsymbol{H}^{s}(\Omega)$ by $(\boldsymbol{w}, \boldsymbol{v})_{s}:=\sum_{i=1}^{d}\left(w_{i}, v_{i}\right)_{H^{s}(\Omega)}$. These spaces have the natural norms $\|\cdot\|_{s}:=\sqrt{(\cdot, \cdot)_{s}}$ and, of course, $\boldsymbol{L}_{2}(\Omega) \equiv \boldsymbol{H}^{0}(\Omega)$. We use $(\cdot, \cdot)$ to denote the inner product on both $L_{2}(\Omega)$ and $\boldsymbol{L}_{2}(\Omega)$ and will introduce additional notation as and when necessary below. In particular, the natural energy space for this problem is given by

$$
\boldsymbol{X}:=\left\{\boldsymbol{v} \in \boldsymbol{H}^{1}(\Omega): \boldsymbol{v}=\mathbf{0} \text { on } \Gamma_{D}\right\},
$$

and we also define the symmetric bilinear forms $a, b: \boldsymbol{X} \times \boldsymbol{X} \rightarrow \mathbb{R}$ by

$$
\begin{aligned}
a(\boldsymbol{\vartheta}, \boldsymbol{v}) & :=\int_{\Omega} D_{i j k l} \varepsilon_{k l}(\boldsymbol{\vartheta}) \varepsilon_{i j}(\boldsymbol{v}) d \Omega, \\
b(\boldsymbol{\vartheta}, \boldsymbol{v}) & :=\gamma_{M}(\varrho \vartheta, \boldsymbol{v})+\gamma_{E} a(\boldsymbol{\vartheta}, \boldsymbol{v})
\end{aligned}
$$

for all $\boldsymbol{\vartheta}, \boldsymbol{v} \in \boldsymbol{X}$. 
It is easy to see that $a(\cdot, \cdot)$ and $b(\cdot, \cdot)$ are continuous on $\boldsymbol{H}^{1}(\Omega)$, but not so easy to see that for a positive constant $c$ we also have $a(\boldsymbol{v}, \boldsymbol{v}) \geqslant c\|\boldsymbol{v}\|_{\boldsymbol{H}^{1}(\Omega)}^{2}$ for all $\boldsymbol{v} \in \boldsymbol{X}$. This coercivity of $a(\cdot, \cdot)$ follows from our requirement that meas $\partial \Omega\left(\Gamma_{D}\right)>0$ in (17), and is a consequence of Korn-type inequalities. If $\Gamma_{D}=\partial \Omega$ this coercivity is easily established but in the more general case a non-trivial technical argument is needed to show that the coercivity results from excluding the possibility of rigid-body translations and rotations. The details of both of these coercivity results are given in, for example, [21, Thm. 3.1; Def. 3.1 and Thm. 3.5], and from them it follows that $(\boldsymbol{X}, a(\cdot, \cdot))$ is a Hilbert space equivalent to $\left(\boldsymbol{H}^{1}(\Omega),(\cdot, \cdot)_{1}\right)$ and with topological dual $\boldsymbol{X}^{\prime}$. We will use the induced energy norm $\|\boldsymbol{v}\|_{\boldsymbol{X}}:=\sqrt{a(\boldsymbol{v}, \boldsymbol{v})}$ extensively below.

Testing (5), integrating by parts, using (10), and imposing $\boldsymbol{w}=\dot{\boldsymbol{u}}$ and each of the internal variable evolution equations, (9), individually in the energy inner product $a(\cdot, \cdot)$ we arrive at the weak problem: find $\boldsymbol{u}, \boldsymbol{w}, \boldsymbol{z}_{1}, \ldots, \boldsymbol{z}_{N_{\varphi}}: I \rightarrow \boldsymbol{X}$ such that,

$$
\begin{gathered}
(\varrho \dot{\boldsymbol{w}}(t), \boldsymbol{v})+a\left(\boldsymbol{u}(t), \varphi_{0} \boldsymbol{v}\right)+b(\boldsymbol{w}(t), \boldsymbol{v})+\sum_{q=1}^{N_{\varphi}} a\left(\boldsymbol{z}_{q}(t), \beta_{q} \boldsymbol{v}\right)=\langle L(t), \boldsymbol{v}\rangle, \\
a\left(\boldsymbol{z}_{q}(t)+\tau_{q} \dot{\boldsymbol{z}}_{q}(t)-\beta_{q} \boldsymbol{w}(t), \boldsymbol{v}\right)=0 \quad \text { for each } q=1, \ldots, N_{\varphi}, \\
a\left(\dot{\boldsymbol{u}}(t), \varphi_{0} \boldsymbol{v}\right)=a\left(\boldsymbol{w}(t), \varphi_{0} \boldsymbol{v}\right)
\end{gathered}
$$

where each in turn holds for all $\boldsymbol{v} \in \boldsymbol{X}$, with $\boldsymbol{u}(0)=\breve{\boldsymbol{u}}, \dot{\boldsymbol{u}}(0)=\breve{\boldsymbol{w}}, \boldsymbol{z}_{q}(0)=\mathbf{0}$ for each $q$, and where $L: I \rightarrow \boldsymbol{X}^{\prime}$ is the time dependent linear form defined by,

$$
\langle L(t), \boldsymbol{v}\rangle:=\int_{\Omega} \boldsymbol{v} \cdot \boldsymbol{f}(t) d \Omega+\oint_{\Gamma_{N}} \boldsymbol{v} \cdot \boldsymbol{g}(t) d \Gamma+\left(\varphi_{0}-\varphi(t)\right) a(\breve{\boldsymbol{u}}, \boldsymbol{v}) \quad \forall \boldsymbol{v} \in \boldsymbol{X} .
$$

Our first (unsurprising) result confirms the dissipativity introduced by the viscoelastic damping terms.

Theorem 2.1 (energy balance, dissipation) We have

$$
\begin{aligned}
& \left\|\varrho^{1 / 2} \boldsymbol{w}(t)\right\|_{0}^{2}+\left\|\varphi_{0}^{1 / 2} \boldsymbol{u}(t)\right\|_{\boldsymbol{X}}^{2}+\sum_{q=1}^{N_{\varphi}}\left\|\tau_{q}^{1 / 2} \boldsymbol{z}_{q}(t)\right\|_{\boldsymbol{X}}^{2}+2 \int_{0}^{t} b(\boldsymbol{w}(s), \boldsymbol{w}(s)) d s \\
& +2 \sum_{q=1}^{N_{\varphi}}\left\|\boldsymbol{z}_{q}\right\|_{L_{2}(0, t ; \boldsymbol{X})}^{2}=2 \int_{0}^{t}\langle L(s), \boldsymbol{w}(s)\rangle d s+\left\|\varrho^{1 / 2} \breve{\boldsymbol{w}}\right\|_{0}^{2}+\left\|\varphi_{0}^{1 / 2} \breve{\boldsymbol{u}}\right\|_{\boldsymbol{X}}^{2}
\end{aligned}
$$

for every $t \in I$. Moreover,

$$
\begin{aligned}
& \left\|\varrho^{1 / 2} \boldsymbol{w}(t)\right\|_{0}^{2}+\frac{1}{2}\left\|\varphi_{0}^{1 / 2} \boldsymbol{u}(t)\right\|_{\boldsymbol{X}}^{2}+\sum_{q=1}^{N_{\varphi}}\left\|\tau_{q}^{1 / 2} \boldsymbol{z}_{q}(t)\right\|_{\boldsymbol{X}}^{2} \\
& \quad+2 \int_{0}^{t} b(\boldsymbol{w}(s), \boldsymbol{w}(s)) d s+2 \sum_{q=1}^{N_{\varphi}}\left\|\boldsymbol{z}_{q}\right\|_{L_{2}(0, t ; \boldsymbol{X})}^{2} \\
& \leqslant 2\left\|\varrho^{1 / 2} \breve{\boldsymbol{w}}\right\|_{0}^{2}+\frac{9}{4}\left\|\varphi_{0}^{1 / 2} \breve{\boldsymbol{u}}\right\|_{\boldsymbol{X}}^{2}+32\left\|\varphi_{0}^{-1 / 2} L\right\|_{L_{\infty}\left(0, t ; \boldsymbol{X}^{\prime}\right)}^{2}+16\left\|\varphi_{0}^{-1 / 2} \dot{L}\right\|_{L_{1}\left(0, t ; \boldsymbol{X}^{\prime}\right)}^{2}
\end{aligned}
$$

also for every $t \in I$. 
Proof. Choose $\boldsymbol{v}=2 \boldsymbol{w}$ in (14), $\boldsymbol{v}=2 \boldsymbol{z}_{q}$ for each $q$ in (15), $\boldsymbol{v}=2 \boldsymbol{u}$ in (16), and then add the results together and note that the terms involving $\sum_{q} a\left(\boldsymbol{w}, \beta_{q} \boldsymbol{z}_{q}\right)$ cancel out. We then integrate by parts and use three Young inequalities with $\epsilon=8$ in each to get,

$$
\begin{aligned}
& 2 \int_{0}^{t}\langle L(s), \boldsymbol{w}(s)\rangle d s=2\langle L(t), \boldsymbol{u}(t)\rangle-2\langle L(0), \breve{\boldsymbol{u}}\rangle-2 \int_{0}^{t}\langle\dot{L}(s), \boldsymbol{u}(s)\rangle d s \\
& \quad \leqslant 2\left\|\varphi_{0}^{-1 / 2} L\right\|_{L_{\infty}\left(0, t ; \boldsymbol{X}^{\prime}\right)}\left\|\varphi_{0}^{1 / 2} \boldsymbol{u}\right\|_{L_{\infty}(0, t ; \boldsymbol{X})}+2\left\|\varphi_{0}^{-1 / 2} L\right\|_{L_{\infty}\left(0, t ; \boldsymbol{X}^{\prime}\right)}\left\|\varphi_{0}^{1 / 2} \breve{\boldsymbol{u}}\right\|_{\boldsymbol{X}} \\
& \quad \quad+2\left\|\varphi_{0}^{-1 / 2} \dot{L}\right\|_{L_{1}\left(0, t ; \boldsymbol{X}^{\prime}\right)}\left\|\varphi_{0}^{1 / 2} \boldsymbol{u}\right\|_{L_{\infty}(0, t ; \boldsymbol{X})}, \\
& \quad \leqslant \frac{1}{8}\left\|\varphi_{0}^{1 / 2} \breve{\boldsymbol{u}}\right\|_{\boldsymbol{X}}^{2}+16\left\|\varphi_{0}^{-1 / 2} L\right\|_{L_{\infty}\left(0, t ; \boldsymbol{X}^{\prime}\right)}^{2}+8\left\|\varphi_{0}^{-1 / 2} \dot{L}\right\|_{L_{1}\left(0, t ; \boldsymbol{X}^{\prime}\right)}^{2}+\frac{1}{4}\left\|\varphi_{0}^{1 / 2} \boldsymbol{u}\right\|_{L_{\infty}(0, t ; \boldsymbol{X})}^{2} .
\end{aligned}
$$

The proof is then completed by using a standard kick-back argument.

30000

Remark 2.2 (the choice of internal variable definition) The result just given in Theorem 2.1 did not require Gronwall's lemma and so is in some sense sharp. In fact the cancellation of the $\sum_{q} a\left(\boldsymbol{w}, \beta_{q} \boldsymbol{z}_{q}\right)$ terms rendered the proof almost trivial, and this is why we used (3) rather than (21) to define the internal variables in (8). In fact we could define internal variables using (21), as in [23], and arrive at ODE's similar to those in (91). On a physical level the approaches are equivalent but, in the latter case, the analogue to (15) will contain $\boldsymbol{u}$ and not $\boldsymbol{w}$ and the cancellation used above will not occur. Similarly high quality stability estimates can still be derived in that case but with considerably more effort, and in the space-time Galerkin framework set forth below, this additional effort seems not to bring additional rewards. On the contrary, it will make the definition of a discrete dual problem, as later in (30), more obscure and impede the duality argument used in the derivation of a priori error bounds.

In the next section we give a space-time finite element approximation of this problem using a continuous Galerkin scheme in space and a discontinuous Galerkin scheme in time (DGCG-FEM).

\section{The discrete scheme}

The finite element spatial discretization is performed in a standard way by generating a family of boundary conforming quasi-uniform meshes indexed by an element-size parameter $h$, and then constructing a corresponding family of standard conforming nodal (Lagrange) finite element spaces, $\boldsymbol{X}^{h} \subset \boldsymbol{X}$, of piecewise polynomials of degree $p \geqslant 1$. We assume that these spaces have the usual approximation property,

$$
\inf _{\boldsymbol{v}^{h} \in \boldsymbol{X}^{h}}\left\{\left\|\boldsymbol{v}-\boldsymbol{v}^{h}\right\|_{0}+h\left\|\boldsymbol{v}-\boldsymbol{v}^{h}\right\|_{1}\right\} \leqslant C h^{p+1}\|\boldsymbol{v}\|_{H^{p+1}(\Omega)}
$$

for all $\boldsymbol{v} \in \boldsymbol{H}^{p+1}(\Omega)$. For the time discretization we choose $N \in \mathbb{N}$, define the time step $k=T / N$ and set $I_{n}=\left(t_{n-1}, t_{n}\right)$ with $t_{n}=n k$. Note that although we could anticipate an adaptive solver and allow the time steps and $\boldsymbol{X}^{h}$ to vary with time by using the same approach as in [12], we don't because we are concerned only with an $a$ priori error analysis and we want to keep the exposition simple. 
We recall the $\boldsymbol{L}_{2}(\Omega)$ and elliptic projections, $P_{0}$ and $P_{\boldsymbol{X}}$, defined by

$$
\left(P_{0} \boldsymbol{v}-\boldsymbol{v}, \boldsymbol{\chi}\right)=0 \quad \text { and } \quad a\left(P_{\boldsymbol{X}} \boldsymbol{v}-\boldsymbol{v}, \boldsymbol{\chi}\right)=0
$$

each for all $\chi \in \boldsymbol{X}^{h}$, and we note from (17) that

$$
\left\|\boldsymbol{v}-P_{0} \boldsymbol{v}\right\|_{0} \leqslant C h^{p+1}\|\boldsymbol{v}\|_{H^{p+1}(\Omega)} \quad \text { and } \quad\left\|\boldsymbol{v}-P_{\boldsymbol{X}} \boldsymbol{v}\right\|_{\boldsymbol{X}} \leqslant C h^{p}\|\boldsymbol{v}\|_{H^{p+1}(\Omega)} .
$$

Our other notation is either standard and/or well known in this context. We define the limits,

$$
v_{n}^{ \pm}=\lim _{\epsilon \downarrow 0} v\left(t_{n} \pm \epsilon\right), \quad \text { the jumps, } \quad \llbracket v \rrbracket_{n}:=v_{n}^{+}-v_{n}^{-},
$$

and the temporally local and global space-time forms

$$
((\cdot, \cdot))_{n}:=\int_{I_{n}}(\cdot, \cdot) d t \quad \text { and } \quad((\cdot, \cdot)):=\sum_{n=1}^{N}((\cdot, \cdot))_{n}
$$

with the obvious extensions to $a((\cdot, \cdot))_{n}$ and $\left\langle\langle\cdot, \cdot\rangle_{n}\right.$ locally, and to $a((\cdot, \cdot))$ and $\langle\langle\cdot, \cdot\rangle$ globally. The fully discrete finite element space is built from the space of temporally discontinuous piecewise polynomials of degree $r \geqslant 0$ which have target space $\boldsymbol{X}^{h}$ :

$$
\mathbb{V}_{n}:=\mathbb{P}_{r}\left(I_{n} ; \boldsymbol{X}^{h}\right) \quad \text { and } \quad \mathbb{V}=\left\{\boldsymbol{v} \in L_{\infty}(0, T ; \boldsymbol{X}):\left.\boldsymbol{v}\right|_{I_{n}} \in \mathbb{V}_{n}\right\}
$$

For convenience below we set $\mathbb{V}_{n}^{\times}:=\mathbb{V}_{n}^{2+N_{\varphi}}$ and $\mathbb{V}^{\times}:=\mathbb{V}^{2+N_{\varphi}}$.

The fully discrete approximation of the problem (14), with (15) and (16), is then: for $n=1, \ldots, N$ in turn, find $\left.\left(\boldsymbol{U}, \boldsymbol{W}, \boldsymbol{Z}_{1}, \ldots\right)\right|_{I_{n}} \in \mathbb{V}_{n}^{\times}$such that,

$$
\begin{aligned}
(\varrho \dot{\boldsymbol{W}}, \boldsymbol{\vartheta}))_{n}+ & \left(\varrho \llbracket \boldsymbol{W} \rrbracket_{n-1}, \boldsymbol{\vartheta}_{n-1}^{+}\right)+a\left(\left(\boldsymbol{U}, \varphi_{0} \boldsymbol{\vartheta}\right)\right)_{n}+b((\boldsymbol{W}, \boldsymbol{\vartheta}))_{n}+\sum_{q=1}^{N_{\varphi}} a\left(\left(\boldsymbol{Z}_{q}, \beta_{q} \boldsymbol{\vartheta}\right)\right)_{n} \\
+ & \sum_{q=1}^{N_{\varphi}} a\left(\left(\boldsymbol{Z}_{q}+\tau_{q} \dot{\boldsymbol{Z}}_{q}-\beta_{q} \boldsymbol{W}, \boldsymbol{\xi}_{q}\right)\right)_{n}+\sum_{q=1}^{N_{\varphi}} a\left(\tau_{q} \llbracket \boldsymbol{Z}_{q} \rrbracket_{n-1}, \boldsymbol{\xi}_{q, n-1}^{+}\right) \\
& \left.+a\left(\dot{\boldsymbol{U}}-\boldsymbol{W}, \varphi_{0} \boldsymbol{\zeta}\right)\right)_{n}+a\left(\llbracket \boldsymbol{U} \rrbracket_{n-1}, \varphi_{0} \boldsymbol{\zeta}_{n-1}^{+}\right)=\langle L, \boldsymbol{\vartheta}\rangle_{n}
\end{aligned}
$$

for all $\left(\boldsymbol{\theta}, \boldsymbol{\zeta}, \boldsymbol{\xi}_{1}, \ldots\right) \in \mathbb{V}_{n}^{\times}$and where we define

$$
\boldsymbol{U}_{0}^{-}:=P_{\boldsymbol{X}} \breve{\boldsymbol{u}} \quad \text { and } \quad \boldsymbol{W}_{0}^{-}:=P_{0} \breve{\boldsymbol{w}},
$$

from (18), and $\boldsymbol{Z}_{q, 0}^{-}=\mathbf{0}$ for each $q$. The discrete analogue of the first part of Theorem 2.1 now follows, for which Remark 2.2 remains relevant. The stability estimate is deferred to later (in Theorem 4.4) where we need it for a discrete dual problem.

Theorem 3.1 (dissipation) There exists a unique solution to (20) such that,

$$
\begin{array}{r}
\left\|\varrho^{1 / 2} \boldsymbol{W}_{n}^{-}\right\|_{0}^{2}+\left\|\varphi_{0}^{1 / 2} \boldsymbol{U}_{n}^{-}\right\|_{\boldsymbol{X}}^{2}+\sum_{q=1}^{N_{\varphi}}\left\|\tau_{q}^{1 / 2} \boldsymbol{Z}_{q, n}^{-}\right\|_{\boldsymbol{X}}^{2}+2 \int_{0}^{t_{n}} b(\boldsymbol{W}, \boldsymbol{W}) d t+2 \sum_{q=1}^{N_{\varphi}}\left\|\boldsymbol{Z}_{q}\right\|_{L_{2}\left(0, t_{n} ; \boldsymbol{X}\right)}^{2} \\
+\sum_{m=1}^{n}\left(\left\|\varrho^{1 / 2} \llbracket \boldsymbol{W} \rrbracket_{m-1}\right\|_{0}^{2}+\left\|\varphi_{0}^{1 / 2} \llbracket \boldsymbol{U} \rrbracket_{m-1}\right\|_{\boldsymbol{X}}^{2}+\sum_{q=1}^{N_{\varphi}}\left\|\tau_{q}^{1 / 2} \llbracket \boldsymbol{Z}_{q} \rrbracket_{m-1}\right\|_{\boldsymbol{X}}^{2}\right) \\
=\left\|\varrho^{1 / 2} \boldsymbol{W}_{0}^{-}\right\|_{0}^{2}+\left\|\varphi_{0}^{1 / 2} \boldsymbol{U}_{0}^{-}\right\|_{\boldsymbol{X}}^{2}+\sum_{q=1}^{N_{\varphi}}\left\|\tau_{q}^{1 / 2} \boldsymbol{Z}_{q, 0}^{-}\right\|_{\boldsymbol{X}}^{2}+2 \int_{0}^{t_{n}}\langle L, \boldsymbol{W}\rangle d t
\end{array}
$$

for every $n \in\{1,2, \ldots, N\}$. 
Proof. Given this result we see that zero initial data, $\boldsymbol{W}_{0}^{-}=\boldsymbol{U}_{0}^{-}=\boldsymbol{Z}_{q, 0}^{-}=\mathbf{0}$ (for each $q$ ), and load, $L=0$, would imply only a trivial discrete solution. It follows that a discrete solution exists and is unique for any given set of these data. It remains only to prove the stated equality and for this we first note the identity

$$
((\varrho \dot{\boldsymbol{W}}, \boldsymbol{W}))_{m}+\left(\llbracket \boldsymbol{W} \rrbracket_{m-1}, \varrho \boldsymbol{W}_{m-1}^{+}\right)=\frac{1}{2}\left\|\varrho^{1 / 2} \boldsymbol{W}_{m}^{-}\right\|_{0}^{2}-\frac{1}{2}\left\|\varrho^{1 / 2} \boldsymbol{W}_{m-1}^{-}\right\|_{0}^{2}+\frac{1}{2}\left\|\varrho^{1 / 2} \llbracket \boldsymbol{W} \rrbracket_{m-1}\right\|_{0}^{2}
$$

along with the analogues for $a\left(\dot{\boldsymbol{U}}, \varphi_{0} \boldsymbol{U}\right)$ and $a\left(\dot{\boldsymbol{Z}}_{q}, \tau_{q} \boldsymbol{Z}_{q}\right)$. Next choose $\left(\boldsymbol{\vartheta}, \boldsymbol{\zeta}, \boldsymbol{\xi}_{q}, \ldots\right)=$ $2\left(\boldsymbol{W}, \boldsymbol{U}, \boldsymbol{Z}_{q}, \ldots\right)$ in (20) , note that the terms involving $a\left(\left(\boldsymbol{Z}_{q}, \beta_{q} \boldsymbol{W}\right)\right)_{m}$ cancel out and then sum over $m=1, \ldots, n$.

roror

This discrete energy balance is consistent with that given in Theorem 2.1 for the exact solution, and we also see clearly the numerical dissipation introduced by the jump terms.

Summing over all time levels, we see that the global formulation of (20) is to find $\left(\boldsymbol{U}, \boldsymbol{W}, \boldsymbol{Z}_{1}, \ldots\right) \in \mathbb{V}^{\times}$such that,

$$
\mathscr{A}\left(\left(\boldsymbol{U}, \boldsymbol{W}, \boldsymbol{Z}_{1}, \ldots\right),\left(\boldsymbol{\vartheta}, \boldsymbol{\zeta}, \boldsymbol{\xi}_{1}, \ldots\right)\right)=\mathscr{L}\left(\left(\boldsymbol{\vartheta}, \boldsymbol{\zeta}, \boldsymbol{\xi}_{1}, \ldots\right)\right) \quad \forall\left(\boldsymbol{\theta}, \boldsymbol{\zeta}, \boldsymbol{\xi}_{1}, \ldots\right) \in \mathbb{V}^{\times}
$$

where the linear form is defined by

$$
\mathscr{L}\left(\left(\boldsymbol{\vartheta}, \boldsymbol{\zeta}, \boldsymbol{\xi}_{1}, \ldots\right)\right)=\left(\boldsymbol{W}_{0}^{-}, \varrho \boldsymbol{\vartheta}_{0}^{+}\right)+a\left(\boldsymbol{U}_{0}^{-}, \varphi_{0} \boldsymbol{\zeta}_{0}^{+}\right)+\langle L, \boldsymbol{\vartheta}\rangle
$$

and the bilinear form by,

$$
\begin{gathered}
\left.\mathscr{A}\left(\left(\boldsymbol{U}, \boldsymbol{W}, \boldsymbol{Z}_{1}, \ldots\right),\left(\boldsymbol{\vartheta}, \boldsymbol{\zeta}, \boldsymbol{\xi}_{1}, \ldots\right)\right)=((\varrho \dot{\boldsymbol{W}}, \boldsymbol{\vartheta}))+a\left(\left(\boldsymbol{U}, \varphi_{0} \boldsymbol{\vartheta}\right)\right)+b(\boldsymbol{W}, \boldsymbol{\vartheta})\right) \\
+a\left(\left(\dot{\boldsymbol{U}}-\boldsymbol{W}, \varphi_{0} \boldsymbol{\zeta}\right)\right)+\sum_{q=1}^{N_{\varphi}} a\left(\left(\boldsymbol{Z}_{q}, \beta_{q} \boldsymbol{\vartheta}\right)\right)+\sum_{q=1}^{N_{\varphi}} a\left(\left(\boldsymbol{Z}_{q}+\tau_{q} \dot{\boldsymbol{Z}}_{q}-\beta_{q} \boldsymbol{W}, \boldsymbol{\xi}_{q}\right)\right) \\
+\sum_{n=1}^{N-1}\left(\left(\llbracket \boldsymbol{W} \rrbracket_{n}, \varrho \boldsymbol{\vartheta}_{n}^{+}\right)+a\left(\llbracket \boldsymbol{U} \rrbracket_{n}, \varphi_{0} \boldsymbol{\zeta}_{n}^{+}\right)+\sum_{q=1}^{N_{\varphi}} a\left(\llbracket \boldsymbol{Z}_{q} \rrbracket_{n}, \tau_{q} \boldsymbol{\xi}_{q, n}^{+}\right)\right) \\
+\left(\boldsymbol{W}_{0}^{+}, \varrho \boldsymbol{\vartheta}_{0}^{+}\right)+a\left(\boldsymbol{U}_{0}^{+}, \varphi_{0} \boldsymbol{\zeta}_{0}^{+}\right)+\sum_{q=1}^{N_{\varphi}} a\left(\boldsymbol{Z}_{q, 0}^{+}, \tau_{q} \boldsymbol{\xi}_{q, 0}^{+}\right) .
\end{gathered}
$$

Noting that $\left(\boldsymbol{U}, \boldsymbol{W}, \boldsymbol{Z}_{1}, \ldots\right)$ can, on recalling (21), be replaced by $\left(\boldsymbol{u}, \boldsymbol{w}, \boldsymbol{z}_{1}, \ldots\right)$ in (22) we obtain the following Galerkin orthogonality relationship

$$
\mathscr{A}\left(\left(\boldsymbol{u}, \boldsymbol{w}, \boldsymbol{z}_{1}, \ldots\right)-\left(\boldsymbol{U}, \boldsymbol{W}, \boldsymbol{Z}_{1}, \ldots\right),\left(\boldsymbol{\vartheta}, \boldsymbol{\zeta}, \boldsymbol{\xi}_{1}, \ldots\right)\right)=0 \quad \forall\left(\boldsymbol{\theta}, \boldsymbol{\zeta}, \boldsymbol{\xi}_{1}, \ldots\right) \in \mathbb{V}^{\times}
$$

In the next section we address the convergence of this scheme.

\section{A priori error estimates}

To give a priori error bounds for the discrete approximation, (20), or (22), of (14), (15) and (16) we make some mostly-standard assumptions regarding regularity and data. The important ones are captured in the following block. 
Assumptions 4.1 (technical assumptions) For the error analysis in this section we restrict to the specific case of piecewise linear polynomial approximation in space and time. As already mentioned we assume that the material coefficients are constant in space and time, that the body is a synchronous linear viscoelastic solid with $0<\varphi_{0} \leqslant 1$, that the domain $\Omega$ is a convex polytope that is exactly represented by the finite element mesh, and also that $\Gamma_{N}=\varnothing$ so that $\boldsymbol{X}=\boldsymbol{H}_{0}^{1}(\Omega)$. We further assume regularity of data and domain sufficient to guarantee that the system (14), (15), (16) has a unique solution $\boldsymbol{u} \in W_{\infty}^{3}\left(I ; \boldsymbol{X} \cap \boldsymbol{H}^{3}(\Omega)\right)$ and we assume elliptic regularity such that for every $\boldsymbol{\ell} \in \boldsymbol{L}_{2}(\Omega)$ the solution, $\boldsymbol{q} \in \boldsymbol{X}$ to the elasticity problem $a(\boldsymbol{q}, \boldsymbol{v})=(\boldsymbol{\ell}, \boldsymbol{v})$ for all $\boldsymbol{v} \in \boldsymbol{X}$ satisfies $\|\boldsymbol{q}\|_{\boldsymbol{H}^{2}(\Omega)} \leqslant C_{e}\|\boldsymbol{\ell}\|_{0}$.

As a consequence of these assumptions and the Riesz representation theorem we may define a linear elasticity analogue of the inverse Laplacian as $\mathcal{G}: \boldsymbol{L}_{2}(\Omega) \rightarrow \boldsymbol{X}$ by the relationship $a(\mathcal{G} \boldsymbol{\ell}, \boldsymbol{v})=(\boldsymbol{\ell}, \boldsymbol{v})$ for all $\boldsymbol{v} \in \boldsymbol{X}$ as well as its discrete analogue $\mathcal{G}_{h}: \boldsymbol{L}_{2}(\Omega) \rightarrow$ $\boldsymbol{X}^{h}$ given by $a\left(\mathcal{G}_{h} \boldsymbol{\ell}, \boldsymbol{v}\right)=(\boldsymbol{\ell}, \boldsymbol{v})$ for all $\boldsymbol{v} \in \boldsymbol{X}^{h}$.

Theorem 4.2 (e.g. [27, Chap. 2]) The map $\mathcal{G}: \boldsymbol{L}_{2}(\Omega) \rightarrow \boldsymbol{X}$ defined above is selfadjoint and positive definite on $\boldsymbol{L}_{2}(\Omega)$. Also, $\mathcal{G}_{h}: \boldsymbol{L}_{2}(\Omega) \rightarrow \boldsymbol{X}^{h}$ is self-adjoint and positive semi-definite on $\boldsymbol{L}_{2}(\Omega)$. Furthermore, there are positive constants, $C, C_{\star}$, such that

$$
\begin{aligned}
\left\|\left(\mathcal{G}-\mathcal{G}_{h}\right) \boldsymbol{\ell}\right\|_{0} & \leqslant C h^{2}\|\boldsymbol{\ell}\|_{0} \\
\left|\left(\mathcal{G}_{h} \boldsymbol{\varkappa}, \boldsymbol{\ell}\right)\right| & \leqslant C\|\boldsymbol{\varkappa}\|_{\boldsymbol{X}^{\prime}}\|\boldsymbol{\ell}\|_{0}, \\
\|\ell\|_{\boldsymbol{X}^{\prime}}^{2} & \leqslant\left|\left(\boldsymbol{\ell}, \mathcal{G}_{h} \boldsymbol{\ell}\right)\right|+C_{\star} h^{2}\|\boldsymbol{\ell}\|_{0}^{2}
\end{aligned}
$$

for all $\boldsymbol{\ell}, \boldsymbol{\varkappa} \in \boldsymbol{L}_{2}(\Omega)$.

Proof. For arbitrary $\boldsymbol{\ell} \in \boldsymbol{L}_{2}(\Omega)$ we have $(\mathcal{G} \boldsymbol{\ell}, \boldsymbol{\varkappa})=a(\mathcal{G} \boldsymbol{\ell}, \mathcal{G} \boldsymbol{\varkappa})=(\boldsymbol{\ell}, \mathcal{G} \boldsymbol{\varkappa})$, as well as $(\mathcal{G} \boldsymbol{\ell}, \boldsymbol{\ell})=\|\mathcal{G} \boldsymbol{\ell}\|_{\boldsymbol{X}}^{2} \geqslant 0$ with $\mathcal{G} \boldsymbol{\ell}=\mathbf{0}$ if and only if $\boldsymbol{\ell}=\mathbf{0}$. Furthermore, by the same reasoning $\left(\mathcal{G}_{h} \boldsymbol{\ell}, \boldsymbol{\varkappa}\right)=a\left(\mathcal{G}_{h} \boldsymbol{\ell}, \mathcal{G}_{h} \boldsymbol{\varkappa}\right)=\left(\boldsymbol{\ell}, \mathcal{G}_{h} \boldsymbol{\varkappa}\right)$ and $\left(\mathcal{G}_{h} \boldsymbol{\ell}, \boldsymbol{\ell}\right)=\left\|\mathcal{G}_{h} \boldsymbol{\ell}\right\|_{\boldsymbol{X}}^{2} \geqslant 0$ for all $\ell \in \boldsymbol{L}_{2}(\Omega)$.

Next, by standard energy and approximation error estimates, followed by the AubinNitsche duality technique we get $\left\|\left(\mathcal{G}-\mathcal{G}_{h}\right) \boldsymbol{\ell}\right\|_{0} \leqslant C h^{2}\|\mathcal{G} \boldsymbol{\ell}\|_{\boldsymbol{H}^{2}(\Omega)}$ and (26) then follows from elliptic regularity.

Notice now that $\left\|\mathcal{G}_{h} \ell\right\|_{\boldsymbol{X}}^{2}=\left(\mathcal{G}_{h} \ell, \ell\right) \leqslant\left\|\mathcal{G}_{h} \ell\right\|_{\boldsymbol{X}}\|\boldsymbol{\ell}\|_{\boldsymbol{X}^{\prime}}$ which gives $\left\|\mathcal{G}_{h} \boldsymbol{\ell}\right\|_{\boldsymbol{X}} \leqslant C\|\boldsymbol{\ell}\|_{0}$ because

$$
\|\boldsymbol{\ell}\|_{\boldsymbol{X}^{\prime}}=\sup _{\boldsymbol{v} \in \boldsymbol{X} \backslash\{0\}} \frac{(\boldsymbol{\ell}, \boldsymbol{v})}{\|\boldsymbol{v}\|_{\boldsymbol{X}}} \leqslant\|\boldsymbol{\ell}\|_{0} \sup _{\boldsymbol{v} \in \boldsymbol{X} \backslash\{0\}} \frac{\|\boldsymbol{v}\|_{0}}{\|\boldsymbol{v}\|_{\boldsymbol{X}}} \leqslant C\|\boldsymbol{\ell}\|_{0}
$$

and therefore $\left|\left(\mathcal{G}_{h} \varkappa, \ell\right)\right| \leqslant\|\boldsymbol{\varkappa}\|_{\boldsymbol{X}^{\prime}}\left\|\mathcal{G}_{h} \boldsymbol{\ell}\right\|_{\boldsymbol{X}} \leqslant C\|\boldsymbol{\varkappa}\|_{\boldsymbol{X}^{\prime}}\|\boldsymbol{\ell}\|_{0}$ as claimed in (27). Lastly, for (28) we notice the isometry $\|\mathcal{G} \boldsymbol{\ell}\|_{\boldsymbol{X}}=\|\boldsymbol{\ell}\|_{\boldsymbol{X}^{\prime}}$ for all $\ell \in \boldsymbol{L}_{2}(\Omega)$ from the Riesz theorem. Therefore, for every $\boldsymbol{\ell} \in \boldsymbol{L}_{2}(\Omega)$

$$
\|\boldsymbol{\ell}\|_{\boldsymbol{X}^{\prime}}^{2}=\|\mathcal{G} \boldsymbol{\ell}\|_{\boldsymbol{X}}^{2}=(\boldsymbol{\ell}, \mathcal{G} \boldsymbol{\ell})=\left(\boldsymbol{\ell}, \mathcal{G}_{h} \ell\right)+\left(\boldsymbol{\ell}, \mathcal{G} \boldsymbol{\ell}-\mathcal{G}_{h} \ell\right)
$$

and, from (26) $),\|\boldsymbol{\ell}\|_{\boldsymbol{X}^{\prime}}^{2} \leqslant\left|\left(\boldsymbol{\ell}, \mathcal{G}_{h} \boldsymbol{\ell}\right)\right|+C h^{2}\|\boldsymbol{\ell}\|_{0}^{2}$ which is (28)$)$. 
To handle the time discretization errors we introduce, piecewise for each $n$, the projection $\left.P_{I}\right|_{I_{n}}: C\left(\bar{I}_{n}\right) \rightarrow \mathbb{P}_{1}\left(I_{n}\right)$ defined by

$$
\left(P_{I} v\right)_{n}^{-}=v_{n}^{-} \quad \text { and } \quad \int_{t_{n-1}}^{t_{n}} v(t)-P_{I} v(t) d t=0 .
$$

We will need the following estimates of the approximation error associated with $P_{I}$ as well as the error associated with the piecewise constant approximation of a function $v \in L_{1}\left(I_{n}\right)$ by its average value $\bar{v}:=\frac{1}{k} \int_{t_{n-1}}^{t_{n}} v(s) d s$. The proofs are standard and are omitted.

Lemma 4.3 If $(\boldsymbol{Y},(\cdot, \cdot))$, with induced norm $\|\cdot\|_{\boldsymbol{Y}}$, is either $\boldsymbol{L}_{2}(\Omega)$ or one of its Hilbert subspaces, then for any $p \in[1, \infty]$ we have $\|\boldsymbol{v}-\overline{\boldsymbol{v}}\|_{L_{p}\left(I_{n} ; \boldsymbol{Y}\right)} \leqslant k\|\boldsymbol{v}\|_{L_{p}\left(I_{n} ; \boldsymbol{Y}\right)}$ and $\left\|\left(I-P_{I}\right) \boldsymbol{v}\right\|_{L_{p}\left(I_{n} ; \boldsymbol{Y}\right)} \leqslant 2 k^{2}\|\ddot{\boldsymbol{v}}\|_{L_{p}\left(I_{n} ; \boldsymbol{Y}\right)}$.

The next step is to introduce a discrete dual backward problem and establish strong stability estimates for its solution. We then use this dual problem to obtain an error representation formula and the error bound will follow from that, the dual stability estimates and approximation results. The discrete dual backward problem is: find $\left(\mathcal{U}, \mathcal{W}, \mathcal{Z}_{1}, \ldots\right) \in \mathbb{V}^{\times}$such that

$$
\mathscr{A}^{*}\left(\left(\mathcal{W}, \mathcal{U}, \mathcal{Z}_{1}, \ldots\right),\left(\boldsymbol{\vartheta}, \boldsymbol{\zeta}, \boldsymbol{\xi}_{1}, \ldots\right)\right)=\mathscr{G}\left(\left(\boldsymbol{\vartheta}, \boldsymbol{\zeta}, \boldsymbol{\xi}_{1}, \ldots\right)\right) \quad \forall\left(\boldsymbol{\theta}, \boldsymbol{\zeta}, \boldsymbol{\xi}_{1}, \ldots\right) \in \mathbb{V}^{\times}
$$

where the linear form (with data $\mathcal{W}_{N}^{+}, \mathcal{U}_{N}^{+}$and $\mathfrak{g}$ to be chosen later) is defined by

$$
\mathscr{G}\left(\left(\boldsymbol{\vartheta}, \boldsymbol{\zeta}, \boldsymbol{\xi}_{1}, \ldots\right)\right)=\left(\mathcal{W}_{N}^{+}, \varrho \boldsymbol{\zeta}_{N}^{-}\right)+a\left(\mathcal{U}_{N}^{+}, \varphi_{0} \boldsymbol{\vartheta}_{N}^{-}\right)+\langle\langle\mathfrak{g}, \boldsymbol{\zeta}\rangle
$$

and the bilinear form is defined by,

$$
\begin{gathered}
\mathscr{A}^{*}\left(\left(\mathcal{W}, \mathcal{U}, \mathcal{Z}_{1}, \ldots\right),\left(\boldsymbol{\vartheta}, \boldsymbol{\zeta}, \boldsymbol{\xi}_{1}, \ldots\right)\right)=-((\varrho \dot{\mathcal{W}}, \boldsymbol{\zeta}))-a\left(\left(\mathcal{U}, \varphi_{0} \boldsymbol{\zeta}\right)\right)+b((\boldsymbol{\zeta}, \mathcal{W})) \\
+a\left(\left(\mathcal{W}-\dot{\mathcal{U}}, \varphi_{0} \boldsymbol{\vartheta}\right)\right)-\sum_{q=1}^{N_{\varphi}} a\left(\left(\mathcal{Z}_{q}, \beta_{q} \boldsymbol{\zeta}\right)\right)+\sum_{q=1}^{N_{\varphi}} a\left(\left(\mathcal{Z}_{q}-\tau_{q} \dot{\mathcal{Z}}_{q}+\beta_{q} \mathcal{W}, \boldsymbol{\xi}_{q}\right)\right) \\
-\sum_{n=1}^{N-1}\left(\left(\llbracket \mathcal{W} \rrbracket_{n}, \varrho \boldsymbol{\zeta}_{n}^{-}\right)+a\left(\llbracket \mathcal{U} \rrbracket_{n}, \varphi_{0} \boldsymbol{\vartheta}_{n}^{-}\right)+\sum_{q=1}^{N_{\varphi}} a\left(\llbracket \mathcal{Z}_{q} \rrbracket_{n}, \tau_{q} \boldsymbol{\xi}_{q, n}^{-}\right)\right) \\
+\left(\mathcal{W}_{N}^{-}, \varrho \boldsymbol{\zeta}_{N}^{-}\right)+a\left(\mathcal{U}_{N}^{-}, \varphi_{0} \boldsymbol{\vartheta}_{N}^{-}\right)+\sum_{q=1}^{N_{\varphi}} a\left(\mathcal{Z}_{q, N}^{-}, \tau_{q} \boldsymbol{\xi}_{q, N}^{-}\right) .
\end{gathered}
$$

If we define $\boldsymbol{X} \rightarrow \boldsymbol{X}^{\prime}$ maps $A$ and $B^{*}$ using the bilinear forms so that $\langle A \boldsymbol{\chi}, \boldsymbol{\theta}\rangle=a(\boldsymbol{\chi}, \boldsymbol{\theta})$ and $\left\langle B^{*} \boldsymbol{\chi}, \boldsymbol{\theta}\right\rangle=b(\boldsymbol{\theta}, \boldsymbol{\chi})$ each for all $\boldsymbol{\theta}, \boldsymbol{\chi} \in \boldsymbol{X}$ then this corresponds to a discrete approximation to a backward problem which in 'strong form', and with $\mathcal{W}=\dot{\mathcal{U}}$, looks like $\varrho \dot{\mathcal{W}}+\varphi_{0} A \mathcal{U}-B^{*} \mathcal{W}+\sum_{q} \beta_{q} A \mathcal{Z}_{q}=-\mathfrak{g}$ and $\tau_{q} \dot{\mathcal{Z}}_{q}-\mathcal{Z}_{q}=\beta_{q} \mathcal{W}$ for each $q$.

Integrating by parts in time and using,

$$
\begin{aligned}
\sum_{n=1}^{N} \int_{t_{n-1}}^{t_{n}}(\varrho \dot{\boldsymbol{\zeta}}, \mathcal{W}) d t & +\sum_{n=1}^{N-1}\left(\varrho \llbracket \boldsymbol{\zeta} \rrbracket_{n}, \mathcal{W}_{n}^{+}\right)+\left(\varrho \boldsymbol{\zeta}_{0}^{+}, \mathcal{W}_{0}^{+}\right) \\
& =\sum_{n=1}^{N} \int_{t_{n-1}}^{t_{n}}(-\varrho \dot{\mathcal{W}}, \boldsymbol{\zeta}) d t+\sum_{n=1}^{N-1}\left(-\varrho \llbracket \mathcal{W} \rrbracket_{n}, \boldsymbol{\zeta}_{n}^{-}\right)+\left(\varrho \mathcal{W}_{N}^{-}, \boldsymbol{\zeta}_{N}^{-}\right),
\end{aligned}
$$


for all $\mathcal{W}$ and $\boldsymbol{\zeta}$ such that $\left.\mathcal{W}\right|_{I_{n}} \in W_{1}^{1}\left(I_{n} ; \boldsymbol{X}\right)$ and $\left.\boldsymbol{\zeta}\right|_{I_{n}} \in W_{1}^{1}\left(I_{n} ; \boldsymbol{X}\right)$ for each $n \in$ $\{1, \ldots, N\}$, with similar results for the terms involving $a\left(\dot{\mathcal{U}}, \varphi_{0} \boldsymbol{\vartheta}\right)$ and $a\left(\dot{\mathcal{Z}}_{q}, \tau_{q} \boldsymbol{\xi}_{q}\right)$, gives that

$$
\mathscr{A}^{*}\left(\left(\mathcal{W}, \mathcal{U}, \mathcal{Z}_{1}, \ldots\right),\left(\boldsymbol{\vartheta}, \boldsymbol{\zeta}, \boldsymbol{\xi}_{1}, \ldots\right)\right)=\mathscr{A}\left(\left(\boldsymbol{\vartheta}, \boldsymbol{\zeta}, \boldsymbol{\xi}_{1}, \ldots\right),\left(\mathcal{W}, \mathcal{U}, \mathcal{Z}_{1}, \ldots\right)\right)
$$

Let $\Pi_{u}, \Pi_{w}, \Pi_{1}, \ldots, \Pi_{N_{\varphi}}: H^{1}(I ; \boldsymbol{X}) \rightarrow \mathbb{V}$ be projections, as yet unspecified. Then, on choosing $\left(\boldsymbol{\theta}, \boldsymbol{\zeta}, \boldsymbol{\xi}_{1}, \ldots\right)=\left(\boldsymbol{U}, \boldsymbol{W}, \boldsymbol{Z}_{1}, \ldots\right)-\left(\Pi_{u} \boldsymbol{u}, \Pi_{w} \boldsymbol{w}, \Pi_{1} \boldsymbol{z}_{1}, \ldots\right)$ in (30), and using (33) and the Galerkin orthogonality in (25), we obtain the error representation formula,

$$
\begin{aligned}
\mathscr{G}\left(\left(\boldsymbol{\vartheta}, \boldsymbol{\zeta}, \boldsymbol{\xi}_{1}, \ldots\right)\right) & =\mathscr{G}\left(\left(\boldsymbol{U}-\Pi_{u} \boldsymbol{u}, \boldsymbol{W}-\Pi_{w} \boldsymbol{w}, \boldsymbol{Z}_{1}-\Pi_{1} \boldsymbol{z}_{1}, \ldots\right)\right) \\
& =\mathscr{A}^{*}\left(\left(\mathcal{W}, \mathcal{U}, \mathcal{Z}_{1}, \ldots\right),\left(\boldsymbol{U}-\Pi_{u} \boldsymbol{u}, \boldsymbol{W}-\Pi_{w} \boldsymbol{w}, \boldsymbol{Z}_{1}-\Pi_{1} \boldsymbol{z}_{1}, \ldots\right)\right) \\
& =\mathscr{A}\left(\left(\boldsymbol{U}-\Pi_{u} \boldsymbol{u}, \boldsymbol{W}-\Pi_{w} \boldsymbol{w}, \boldsymbol{Z}_{1}-\Pi_{1} \boldsymbol{z}_{1}, \ldots\right),\left(\mathcal{W}, \mathcal{U}, \mathcal{Z}_{1}, \ldots\right)\right) \\
& =\mathscr{A}\left(\left(\boldsymbol{u}-\Pi_{u} \boldsymbol{u}, \boldsymbol{w}-\Pi_{w} \boldsymbol{w}, \boldsymbol{z}_{1}-\Pi_{1} \boldsymbol{z}_{1}, \ldots\right),\left(\mathcal{W}, \mathcal{U}, \mathcal{Z}_{1}, \ldots\right)\right)
\end{aligned}
$$

The terms $\left(\boldsymbol{u}-\Pi_{u} \boldsymbol{u}, \boldsymbol{w}-\Pi_{w} \boldsymbol{w}, \boldsymbol{z}_{1}-\Pi_{1} \boldsymbol{z}_{1}, \ldots\right)$ on the right can be bounded by approximation results and then once the terms involving $\left(\mathcal{W}, \mathcal{U}, \mathcal{Z}_{1}, \ldots\right)$ are bounded by the data in $\mathscr{G}$, and suitable choices for those data are made, we will obtain an a priori estimate for $\boldsymbol{U}-\Pi_{u} \boldsymbol{u}$ and $\boldsymbol{W}-\Pi_{w} \boldsymbol{w}$. The estimates for $\boldsymbol{u}-\boldsymbol{U}$ and $\boldsymbol{w}-\boldsymbol{W}$ then follow from more approximation estimates and the triangle inequality.

We begin by determining an analogue of Theorem 3.1, and derive stability estimates for the discrete dual problem where the final values of the dual internal variables are zero. In this and below it is to be understood that the temporal norms of time derivatives are 'broken' so that $\|\dot{\mathcal{W}}\|_{L_{p}\left(I_{; \cdot}\right)}=\left(\sum_{n}\|\dot{\mathcal{W}}\|_{L_{p}\left(I_{n} ; \cdot\right)}^{p}\right)^{1 / p}$ with the 'max $\{\ldots\}$ ' modification for $p=\infty$.

Theorem 4.4 (discrete dual stability) Let Assumptions 4.1 hold and then, with $\mathcal{Z}_{q}(T)=\mathbf{0}$ for each $q$, there exists a unique solution to (30) that satisfies

$$
\begin{array}{r}
\left.\left\|\varrho^{1 / 2} \mathcal{W}_{n}^{+}\right\|_{0}^{2}+\left\|\varphi_{0}^{1 / 2} \mathcal{U}_{n}^{+}\right\|_{\boldsymbol{X}}^{2}+\sum_{q=1}^{N_{\varphi}}\left\|\tau_{q}^{1 / 2} \mathcal{Z}_{q, n}^{+}\right\|_{\boldsymbol{X}}^{2}+2 \int_{t_{n}}^{T} b(\mathcal{W}, \mathcal{W}) d t+2 \sum_{q=1}^{N_{\varphi}}\left\|\mathcal{Z}_{q}\right\|_{L_{2}\left(t_{n}, T ; \boldsymbol{X}\right.}^{2}\right) \\
+\sum_{m=n+1}^{N}\left(\left\|\varrho^{1 / 2} \llbracket \mathcal{W} \rrbracket_{m}\right\|_{0}^{2}+\left\|\varphi_{0}^{1 / 2} \llbracket \mathcal{U} \rrbracket_{m}\right\|_{\boldsymbol{X}}^{2}+\sum_{q=1}^{N_{\varphi}}\left\|\tau_{q}^{1 / 2} \llbracket \mathcal{Z}_{q} \rrbracket_{m}\right\|_{\boldsymbol{X}}^{2}\right) \\
=\left\|\varrho^{1 / 2} \mathcal{W}_{N}^{+}\right\|_{0}^{2}+\left\|\varphi_{0}^{1 / 2} \mathcal{U}_{N}^{+}\right\|_{\boldsymbol{X}}^{2}+2 \int_{t_{n}}^{T}\langle\mathfrak{g}, \mathcal{W}\rangle d t
\end{array}
$$

for every $n=0,1, \ldots, N-1$. If in addition $\mathfrak{g}=0$ and $h \leqslant c_{T} k$ for a positive constant $c_{T}$ then

$$
\begin{gathered}
\left\|\varrho^{1 / 2} \mathcal{W}\right\|_{L_{\infty}\left(I ; \boldsymbol{L}_{2}(\Omega)\right)}^{2}+\left\|\varrho^{1 / 2} \dot{\mathcal{W}}\right\|_{L_{p}\left(I ; \boldsymbol{X}^{\prime}\right)}^{2}+\left\|\varphi_{0}^{1 / 2} \mathcal{U}\right\|_{L_{\infty}\left(I_{n} ; \boldsymbol{X}\right)}^{2}+\left\|\varphi_{0}^{1 / 2} \dot{\mathcal{U}}\right\|_{L_{\infty}\left(I ; \boldsymbol{L}_{2}(\Omega)\right)}^{2} \\
\quad+\sum_{q=1}^{N_{\varphi}}\left\|\tau_{q}^{1 / 2} \dot{\mathcal{Z}}_{q}\right\|_{L_{\infty}(I ; \boldsymbol{X})}^{2} \leqslant C T^{2 / p}\left(1+c_{T}^{2}\right)\left(\left\|\varrho^{1 / 2} \mathcal{W}_{N}^{+}\right\|_{0}^{2}+\left\|\varphi_{0}^{1 / 2} \mathcal{U}_{N}^{+}\right\|_{\boldsymbol{X}}^{2}\right)
\end{gathered}
$$

for a constant $C$ independent of $T, h$ and $k$ and where we can choose $p=\infty$ if $\gamma_{E}=0$ in (13) and $p=2$ if $\gamma_{E}>0$. 
Proof. Notice that if the data, $\mathcal{W}_{N}^{+}, \mathcal{U}_{N}^{+}$and $\mathfrak{g}$, are zero in the discrete dual problem then the first claim of the theorem provides uniqueness of solution, and existence then follows. To prove this equality, in analogy to the proof of Theorem 3.1, we choose in (30) $\left(\boldsymbol{\vartheta}, \boldsymbol{\zeta}, \boldsymbol{\xi}_{1}, \ldots\right)=\left(\mathcal{U}, \mathcal{W}, \mathcal{Z}_{1}, \ldots\right)$ on $\left(t_{n}, T\right)$, and zero on $\left(0, t_{n}\right)$, for an arbitrary $n=0,1, \ldots, N-1$, and noting that

$$
\begin{aligned}
-\sum_{m=n+1}^{N} \int_{t_{m-1}}^{t_{m}} \frac{1}{2} \frac{d}{d t}\left\|\varrho^{1 / 2} \mathcal{W}\right\|_{0}^{2} d t-\sum_{m=n+1}^{N-1}\left(\mathcal{W}_{m}^{+}-\mathcal{W}_{m}^{-}, \varrho \mathcal{W}_{m}^{-}\right)+\left\|\varrho^{1 / 2} \mathcal{W}_{N}^{-}\right\|_{0}^{2} \\
=\frac{1}{2} \sum_{m=n+1}^{N}\left\|\varrho^{1 / 2} \mathcal{W}_{m}^{-}\right\|_{0}^{2}+\frac{1}{2} \sum_{m=n+1}^{N}\left\|\varrho^{1 / 2} \mathcal{W}_{m-1}^{+}\right\|_{0}^{2}-\sum_{m=n+1}^{N-1}\left(\mathcal{W}_{m}^{+}, \varrho \mathcal{W}_{m}^{-}\right), \\
=\frac{1}{2} \sum_{m=n}^{N-1}\left\|\varrho^{1 / 2} \mathcal{W}_{m}^{+}\right\|_{0}^{2}-\frac{1}{2} \sum_{m=n+1}^{N}\left\|\varrho^{1 / 2} \mathcal{W}_{m}^{+}\right\|_{0}^{2}+\left(\mathcal{W}_{N}^{+}, \varrho \mathcal{W}_{N}^{-}\right) \\
\quad+\frac{1}{2} \sum_{m=n+1}^{N}\left[\left(\mathcal{W}_{m}^{-}, \varrho \mathcal{W}_{m}^{-}\right)+\left(\mathcal{W}_{m}^{+}, \varrho \mathcal{W}_{m}^{+}\right)-2\left(\mathcal{W}_{m}^{+}, \varrho \mathcal{W}_{m}^{-}\right)\right]
\end{aligned}
$$

which leads eventually to

$$
\begin{aligned}
& -\sum_{m=n+1}^{N} \int_{t_{m-1}}^{t_{m}}(\varrho \dot{\mathcal{W}}, \mathcal{W}) d t-\sum_{m=n+1}^{N-1}\left(\llbracket \mathcal{W} \rrbracket_{m}, \varrho \mathcal{W}_{m}^{-}\right)+\left(\varrho \mathcal{W} \mathcal{W}_{N}^{-}, \mathcal{W}_{N}^{-}\right) \\
& \quad=\frac{1}{2}\left\|\varrho^{1 / 2} \mathcal{W}_{n}^{+}\right\|_{0}^{2}-\frac{1}{2}\left\|\varrho^{1 / 2} \mathcal{W}_{N}^{+}\right\|_{0}^{2}+\frac{1}{2} \sum_{m=n+1}^{N}\left\|\varrho^{1 / 2} \llbracket \mathcal{W} \rrbracket_{m}\right\|_{0}^{2}+\left(\varrho \mathcal{W}_{N}^{+}, \mathcal{W}_{N}^{-}\right)
\end{aligned}
$$

along with the analogues for $a\left(\dot{\mathcal{U}}, \varphi_{0} \mathcal{U}\right)$ and $a\left(\dot{\mathcal{Z}}_{q}, \tau_{q} \mathcal{Z}_{q}\right)$, then gives the first part of the theorem once we set $\mathcal{Z}_{q, N}^{+}=\mathcal{Z}_{q, N}^{-}=\mathbf{0}$.

Next, in (30) we choose $\left.\boldsymbol{\zeta}\right|_{I_{n}}=\left(t_{n}-t\right) \mathcal{G}_{h} \dot{\mathcal{W}}$ to obtain after recalling that $\beta_{q}=\left(\varphi_{q} \tau_{q}\right)^{1 / 2}$, using (28) and then (27) with several applications of Hölder's and Young's inequalities,

$$
\begin{gathered}
\left\|\varrho^{1 / 2} \dot{\mathcal{W}}\right\|_{\boldsymbol{X}^{\prime}}^{2} \leqslant C \\
\gamma_{E}^{2} k^{-2 / p}\|\mathcal{W}\|_{L_{p}\left(I_{n} ; \boldsymbol{X}\right)}^{2}+C h^{2}\left\|\varrho^{1 / 2} \dot{\mathcal{W}}\right\|_{0}^{2}+C\left\|\varrho^{1 / 2} \mathcal{W}\right\|_{L_{\infty}\left(I_{n} ; \boldsymbol{L}_{2}(\Omega)\right)}^{2} \\
+C\left\|\varphi_{0}^{1 / 2} \mathcal{U}\right\|_{L_{\infty}\left(I_{n} ; \boldsymbol{X}\right)}^{2}+C \sum_{q=1}^{N_{\varphi}}\left\|\tau_{q}^{1 / 2} \mathcal{Z}_{q}\right\|_{L_{\infty}\left(I_{n} ; \boldsymbol{X}\right)}^{2}
\end{gathered}
$$

for every $p \in[1, \infty]$. If there is no stiffness term in the Rayleigh damping then $\gamma_{E}=0$ and this estimate is sufficient for our needs, but if $\gamma_{E} \neq 0$ then we need to eliminate the $k^{-2 / p}$ term on the right. To do this we take $p=2$ and obtain, in the general case,

$$
\begin{gathered}
\left\|\varrho^{1 / 2} \dot{\mathcal{W}}\right\|_{L_{p}\left(I ; \boldsymbol{X}^{\prime}\right)}^{2} \leqslant C T^{2 / p} \max _{1 \leqslant n \leqslant N}\left\{h^{2}\left\|\varrho^{1 / 2} \dot{\mathcal{W}}\right\|_{L_{\infty}\left(I_{n} ; \boldsymbol{L}_{2}(\Omega)\right)}^{2}+\left\|\varrho^{1 / 2} \mathcal{W}\right\|_{L_{\infty}\left(I_{n} ; \boldsymbol{L}_{2}(\Omega)\right)}^{2}\right. \\
\left.\quad+\left\|\varphi_{0}^{1 / 2} \mathcal{U}\right\|_{L_{\infty}\left(I_{n} ; \boldsymbol{X}\right)}^{2}+\sum_{q=1}^{N_{\varphi}}\left\|\tau_{q}^{1 / 2} \mathcal{Z}_{q}\right\|_{L_{\infty}\left(I_{n} ; \boldsymbol{X}\right)}^{2}\right\}+C \gamma_{E} \int_{0}^{T} b(\mathcal{W}, \mathcal{W}) d t
\end{gathered}
$$

with $p=2$ when $\gamma_{E}>0$ and $p=\infty$ when $\gamma_{E}=0$. Noting now that on each $I_{n}$ we have $\left.\mathcal{W}\right|_{I_{n}}=k^{-1}\left(t_{n}-t\right) \mathcal{W}_{n-1}^{+}+k^{-1}\left(t-t_{n-1}\right) \mathcal{W}_{n}^{-}$and so on, we can obtain,

$$
\begin{gathered}
k\left\|\varrho^{1 / 2} \dot{\mathcal{W}}\right\|_{L_{\infty}\left(I_{n} ; \boldsymbol{L}_{2}(\Omega)\right)}+\left\|\varrho^{1 / 2} \mathcal{W}\right\|_{L_{\infty}\left(I_{n} ; \boldsymbol{L}_{2}(\Omega)\right)} \leqslant\left\|\varrho^{1 / 2} \mathcal{W}_{n}^{+}\right\|_{0} \\
+\left\|\varrho^{1 / 2} \mathcal{W}_{n-1}^{+}\right\|_{0}+\left\|\varrho^{1 / 2} \llbracket \mathcal{W} \rrbracket_{n}\right\|_{0}
\end{gathered}
$$


with similar results for $\mathcal{U}$ and $\mathcal{W}$ in the $\boldsymbol{X}$ norm, and these imply,

$$
\begin{gathered}
\left\|\varphi_{0}^{1 / 2} \mathcal{U}\right\|_{L_{\infty}\left(I_{n} ; \boldsymbol{X}\right)}^{2}+\left\|\varrho^{1 / 2} \mathcal{W}\right\|_{L_{\infty}\left(I ; \boldsymbol{L}_{2}(\Omega)\right)}^{2}+\left\|\varrho^{1 / 2} \dot{\mathcal{W}}\right\|_{L_{p}\left(I ; \boldsymbol{X}^{\prime}\right)}^{2} \leqslant C \gamma_{E} \int_{0}^{T} b(\mathcal{W}, \mathcal{W}) \\
+C T^{2 / p} \max _{1 \leqslant n \leqslant N}\left\{\left(1+k^{-2} h^{2}\right)\left(\left\|\varrho^{1 / 2} \mathcal{W}_{n}^{+}\right\|_{0}^{2}+\left\|\varrho^{1 / 2} \mathcal{W}_{n-1}^{+}\right\|_{0}^{2}+\left\|\varrho^{1 / 2} \llbracket \mathcal{W} \rrbracket_{n}\right\|_{0}^{2}\right)\right. \\
+\left\|\varphi_{0}^{1 / 2} \mathcal{U}_{n}^{+}\right\|_{\boldsymbol{X}}^{2}+\left\|\varphi_{0}^{1 / 2} \mathcal{U}_{n-1}^{+}\right\|_{\boldsymbol{X}}^{2}+\left\|\varphi_{0}^{1 / 2} \llbracket \mathcal{U} \rrbracket_{n}\right\|_{\boldsymbol{X}}^{2} \\
\left.+\sum_{q=1}^{N_{\varphi}}\left(\left\|\tau_{q}^{1 / 2} \mathcal{Z}_{q, n}^{+}\right\|_{\boldsymbol{X}}^{2}+\left\|\tau_{q}^{1 / 2} \mathcal{Z}_{q, n-1}^{+}\right\|_{\boldsymbol{X}}^{2}+\left\|\tau_{q}^{1 / 2} \llbracket \mathcal{Z}_{q} \rrbracket_{n}\right\|_{\boldsymbol{X}}^{2}\right)\right\} .
\end{gathered}
$$

Returning to (30) with, this time, $\left.\boldsymbol{\vartheta}\right|_{I_{n}}=\varphi_{0}^{-1}\left(t_{n}-t\right) \mathcal{G}_{h} \dot{\mathcal{U}}$ and $\boldsymbol{\vartheta}=\mathbf{0}$ on $I \backslash I_{n}$ we get,

$$
\left\|\varphi_{0}^{1 / 2} \dot{\mathcal{U}}\right\|_{L_{\infty}\left(I_{n} ; \boldsymbol{L}_{2}(\Omega)\right)} \leqslant C\left(\left\|\varrho^{1 / 2} \mathcal{W}_{n}^{+}\right\|_{0}+\left\|\varrho^{1 / 2} \mathcal{W}_{n-1}^{+}\right\|_{0}+\left\|\varrho^{1 / 2} \llbracket \mathcal{W} \rrbracket_{n}\right\|_{0}\right)
$$

In a similar way, with $\left.\boldsymbol{\xi}_{q}\right|_{I_{n}}=\left(t_{n}-t\right) \mathcal{G}_{h} \dot{\mathcal{Z}}_{q}$ in (30) and zero elsewhere we get,

$$
\frac{1}{2} \sum_{q=1}^{N_{\varphi}}\left\|\tau_{q}^{1 / 2} \dot{\mathcal{Z}}_{q}\right\|_{0}^{2} \leqslant \sum_{q=1}^{N_{\varphi}}\left\|\tau_{q}^{-1 / 2} \mathcal{Z}_{q}\right\|_{L_{\infty}\left(I_{n} ; \boldsymbol{L}_{2}(\Omega)\right)}^{2}+\frac{\varphi(0)-\varphi_{0}}{\varrho}\left\|\varrho^{1 / 2} \mathcal{W}\right\|_{L_{\infty}\left(I_{n} ; \boldsymbol{L}_{2}(\Omega)\right)}^{2}
$$

and, therefore, on $I_{n}$ (using $\left.\frac{1}{3}(a+b+c)^{2} \leqslant a^{2}+b^{2}+c^{2}\right)$,

$$
\begin{aligned}
\frac{1}{6} \sum_{q=1}^{N_{\varphi}}\left\|\tau_{q}^{1 / 2} \dot{\mathcal{Z}}_{q}\right\|_{0}^{2} & \leqslant \sum_{q=1}^{N_{\varphi}}\left(\left\|\tau_{q}^{-1 / 2} \mathcal{Z}_{q, n}^{+}\right\|_{0}^{2}+\left\|\tau_{q}^{-1 / 2} \mathcal{Z}_{q, n-1}^{+}\right\|_{0}^{2}+\left\|\tau_{q}^{-1 / 2} \llbracket \mathcal{Z}_{q} \rrbracket_{n}\right\|_{0}^{2}\right) \\
& +\left(\frac{\varphi(0)-\varphi_{0}}{\varrho}\right)\left(\left\|\varrho^{1 / 2} \mathcal{W}_{n}^{+}\right\|_{0}^{2}+\left\|\varrho^{1 / 2} \mathcal{W}_{n-1}^{+}\right\|_{0}^{2}+\left\|\varrho^{1 / 2} \llbracket \mathcal{W} \rrbracket_{n}\right\|_{0}^{2}\right)
\end{aligned}
$$

Assembling these estimates and recalling the first claim of the theorem then completes the proof.

roror

For the linear elasticity operator we can introduce the map $\Delta: \boldsymbol{X} \rightarrow \boldsymbol{L}_{2}(\Omega)$ which is well defined for every $\boldsymbol{v} \in \boldsymbol{H}^{2}(\Omega) \cap \boldsymbol{H}_{0}^{1}(\Omega)$ by $(\Delta \boldsymbol{v}, \boldsymbol{\vartheta})=-a(\boldsymbol{v}, \boldsymbol{\vartheta})$ for all $\boldsymbol{\vartheta} \in \boldsymbol{X}$. We can now give the error bound.

Theorem 4.5 (a priori error bound) Let Assumptions 4.1 hold and in addition assume that $h \leqslant c_{T} k$ for a positive constant $c_{T}$. Then

$$
\left\|\varrho^{1 / 2}(\boldsymbol{w}-\boldsymbol{W})_{N}^{-}\right\|_{0}+\left\|\varphi_{0}^{1 / 2}(\boldsymbol{u}-\boldsymbol{U})_{N}^{-}\right\|_{\boldsymbol{X}} \leqslant C T^{1 / 2+1 / p} \mathscr{R}(\boldsymbol{u})\left(h+k^{3}+k^{-1 / 2} h^{2}\right)
$$

for a constant $C$, dependent on data, but independent of $T, h$ and $k$ and where

$$
\begin{gathered}
\mathscr{R}(\boldsymbol{u})=\|\boldsymbol{u}\|_{W_{r}^{1}\left(I ; \boldsymbol{H}^{2}(\Omega)\right)}+\|\boldsymbol{u}\|_{W_{1}^{1}\left(I ; \boldsymbol{H}^{2}(\Omega)\right)}+\|\boldsymbol{u}\|_{W_{\infty}^{1}\left(I ; \boldsymbol{H}^{2}(\Omega)\right)} \\
+\|\boldsymbol{u}\|_{W_{1}^{3}\left(I ; \boldsymbol{H}^{2}(\Omega)\right)}+\|\boldsymbol{u}\|_{W_{r}^{3}\left(I ; \boldsymbol{H}^{3}(\Omega)\right)} .
\end{gathered}
$$

In this bound we can take $p=\infty$ if $\gamma_{E}=0$ in (13) and $p=2$ if $\gamma_{E}>0$. 
Proof. From the error representation formula, (34), (33) and (32) we have,

$$
\begin{aligned}
\left(\varrho \mathcal{W}_{N}^{+},\right. & \left.\left(\boldsymbol{W}-\Pi_{w} \boldsymbol{w}\right)_{N}^{-}\right)+a\left(\varphi_{0} \mathcal{U}_{N}^{+},\left(\boldsymbol{U}-\Pi_{u} \boldsymbol{u}\right)_{N}^{-}\right)+\left\langle\left\langle\mathfrak{g}, \boldsymbol{W}-\Pi_{w} \boldsymbol{w}\right\rangle\right) \\
& =\mathscr{G}\left(\left(\boldsymbol{U}-\Pi_{u} \boldsymbol{u}, \boldsymbol{W}-\Pi_{w} \boldsymbol{w}, \boldsymbol{Z}_{1}-\Pi_{1} \boldsymbol{z}_{1}, \ldots\right)\right) \\
& =\mathscr{A}\left(\left(\boldsymbol{u}-\Pi_{u} \boldsymbol{u}, \boldsymbol{w}-\Pi_{w} \boldsymbol{w}, \boldsymbol{z}_{1}-\Pi_{1} \boldsymbol{z}_{1}, \ldots\right),\left(\mathcal{W}, \mathcal{U}, \mathcal{Z}_{1}, \ldots\right)\right) \\
& =\mathscr{A}^{*}\left(\left(\mathcal{W}, \mathcal{U}, \mathcal{Z}_{1}, \ldots\right),\left(\boldsymbol{u}-\Pi_{u} \boldsymbol{u}, \boldsymbol{w}-\Pi_{w} \boldsymbol{w}, \boldsymbol{z}_{1}-\Pi_{1} \boldsymbol{z}_{1}, \ldots\right)\right)
\end{aligned}
$$

and so

$$
\begin{aligned}
&\left.\left(\varrho \mathcal{W}_{N}^{+},\left(\boldsymbol{W}-\Pi_{w} \boldsymbol{w}\right)_{N}^{-}\right)+a\left(\varphi_{0} \mathcal{U}_{N}^{+},\left(\boldsymbol{U}-\Pi_{u} \boldsymbol{u}\right)_{N}^{-}\right)+\left\langle\mathfrak{g}, \boldsymbol{W}-\Pi_{w} \boldsymbol{w}\right\rangle\right) \\
&\left.=-\left(\varrho \mathcal{\mathcal { W }}, \boldsymbol{w}-\Pi_{w} \boldsymbol{w}\right)\right)-a\left(\mathcal{U}, \varphi_{0}\left(\boldsymbol{w}-\Pi_{w} \boldsymbol{w}\right)\right)+b\left(\left(\boldsymbol{w}-\Pi_{w} \boldsymbol{w}, \mathcal{W}\right)\right) \\
& \quad+a\left(\left(\mathcal{W}-\dot{\mathcal{U}}, \varphi_{0}\left(\boldsymbol{u}-\Pi_{u} \boldsymbol{u}\right)\right)\right)-\sum_{q=1}^{N_{\varphi}} a\left(\left(\beta_{q} \mathcal{Z}_{q}, \boldsymbol{w}-\Pi_{w} \boldsymbol{w}\right)\right) \\
& \quad-\sum_{n=1}^{N-1}\left(\llbracket \mathcal{W} \rrbracket_{n}, \varrho\left(\boldsymbol{w}-\Pi_{w} \boldsymbol{w}\right)_{n}^{-}\right)+\left(\mathcal{W}_{N}^{-}, \varrho\left(\boldsymbol{w}-\Pi_{w} \boldsymbol{w}\right)_{N}^{-}\right) \\
& \quad-\sum_{n=1}^{N-1} a\left(\llbracket \mathcal{U} \rrbracket_{n}, \varphi_{0}\left(\boldsymbol{u}-\Pi_{u} \boldsymbol{u}\right)_{n}^{-}\right)+a\left(\mathcal{U}_{N}^{-}, \varphi_{0}\left(\boldsymbol{u}-\Pi_{u} \boldsymbol{u}\right)_{N}^{-}\right) \\
&+\sum_{q=1}^{N_{\varphi}} a\left(\left(\mathcal{Z}_{q}+\beta_{q} \mathcal{W}, \boldsymbol{z}_{q}-\Pi_{q} \boldsymbol{z}_{q}\right)\right)-\sum_{q=1}^{N_{\varphi}} a\left(\left(\tau_{q} \dot{\mathcal{Z}}_{q}, \boldsymbol{z}_{q}-\Pi_{q} \boldsymbol{z}_{q}\right)\right) \\
&-\sum_{n=1}^{N-1} \sum_{q=1}^{N_{\varphi}} a\left(\llbracket \mathcal{Z}_{q} \rrbracket_{n}, \tau_{q}\left(\boldsymbol{z}_{q}-\Pi_{q} \boldsymbol{z}_{q}\right)_{n}^{-}\right)+\sum_{q=1}^{N_{\varphi}} a\left(\mathcal{Z}_{q, N}^{-}, \tau_{q}\left(\boldsymbol{z}_{q}-\Pi_{q} \boldsymbol{z}_{q}\right)_{N}^{-}\right) \\
&= \sum_{j=1}^{13} \mathscr{E}_{j}
\end{aligned}
$$

with obvious notation. Recalling $P_{\boldsymbol{X}}$ in (18) and $P_{I}$ in (29), we choose $\Pi_{u}=\Pi_{w}=$ $\Pi_{q}=\Pi$ for $I-\Pi=\left(I-P_{\boldsymbol{X}}\right)+\left(I-P_{I}\right) P_{\boldsymbol{X}}$ and then, with either $(p, r)=(2,2)$ or $(p, r)=(\infty, 1)$ in the following Hölder inqualities, we take the error representation term-by-term to get first that,

$$
\begin{aligned}
\mathscr{E}_{1} & =-\left(\left(\varrho \dot{\mathcal{W}}, \boldsymbol{w}-P_{\boldsymbol{X}} \boldsymbol{w}\right)\right)-\left(\left(\varrho \dot{\mathcal{W}}, P_{\boldsymbol{X}} \boldsymbol{w}-P_{I} P_{\boldsymbol{X}} \boldsymbol{w}\right)\right)=-\left(\left(\varrho \dot{\mathcal{W}},\left(I-P_{\boldsymbol{X}}\right) \boldsymbol{w}\right)\right), \\
\Longrightarrow\left|\mathscr{E}_{1}\right| & \leqslant\left\|\varrho^{1 / 2}\left(I-P_{\boldsymbol{X}}\right) \dot{\boldsymbol{u}}\right\|_{L_{r}(I ; \boldsymbol{X})}\left\|\varrho^{1 / 2} \dot{\mathcal{W}}\right\|_{L_{p}\left(I ; \boldsymbol{X}^{\prime}\right)},
\end{aligned}
$$

and then second that,

$$
\begin{aligned}
\mathscr{E}_{2} & =-a\left(\left(\varphi_{0} \mathcal{U}, \boldsymbol{w}-P_{\boldsymbol{X}} \boldsymbol{w}\right)\right)-a\left(\left(\varphi_{0} \mathcal{U}, P_{\boldsymbol{X}} \boldsymbol{w}-P_{I} P_{\boldsymbol{X}} \boldsymbol{w}\right)\right), \\
& =\sum_{n=1}^{N} \int_{t_{n-1}}^{t_{n}} a\left(\overline{\mathcal{U}}-\mathcal{U}, \varphi_{0}\left(I-P_{I}\right) P_{\boldsymbol{X}} \boldsymbol{w}\right) d t
\end{aligned}
$$

where we introduced the average of $\mathcal{U}$ by virtue of the definition (29) of $P_{I}$. Now, from Lemma 4.3, with $t \in I_{n}$,

$$
\begin{aligned}
\left.a\left(\overline{\mathcal{U}}-\mathcal{U}(t), \varphi_{0}\left(I-P_{I}\right) P_{\boldsymbol{X}} \boldsymbol{w}(t)\right)\right) & =\left(\frac{\varphi_{0}}{k} \int_{t_{n-1}}^{t_{n}} \int_{t}^{s} \dot{\mathcal{U}}(\eta) d \eta d s,\left(I-P_{I}\right) \Delta \boldsymbol{w}(t)\right) \\
& \leqslant \varphi_{0}^{1 / 2} k\left\|\varphi_{0}^{1 / 2} \dot{\mathcal{U}}\right\|_{L_{\infty}\left(I_{n} ; \boldsymbol{L}_{2}(\Omega)\right)}\left\|\left(I-P_{I}\right) \Delta \boldsymbol{w}(t)\right\|_{0} \\
& \leqslant 2 \varphi_{0}^{1 / 2} k^{3}\left\|\varphi_{0}^{1 / 2} \dot{\mathcal{U}}\right\|_{L_{\infty}\left(I_{n} ; \boldsymbol{L}_{2}(\Omega)\right)}\|\Delta \ddot{\boldsymbol{w}}\|_{L_{1}\left(I_{n} ; \boldsymbol{L}_{2}(\Omega)\right)}
\end{aligned}
$$


where we recalled that for $\boldsymbol{\vartheta} \in \boldsymbol{X}^{h}$ we have $a\left(\boldsymbol{\vartheta}, P_{\boldsymbol{X}} \boldsymbol{w}\right)=-(\boldsymbol{\vartheta}, \Delta \boldsymbol{w})$ and also noted that $a\left(P_{I} P_{\boldsymbol{X}} \boldsymbol{w}, \boldsymbol{\vartheta}\right)=-\left(P_{I} \Delta \boldsymbol{w}, \boldsymbol{\vartheta}\right)$. Therefore

$$
\left|\mathscr{E}_{2}\right| \leqslant 2 \varphi_{0}^{1 / 2} k^{3}\left\|\varphi_{0}^{1 / 2} \dot{\mathcal{U}}\right\|_{L_{\infty}\left(I ; \boldsymbol{L}_{2}(\Omega)\right)}\|\Delta \dddot{\boldsymbol{u}}\|_{L_{1}\left(I ; \boldsymbol{L}_{2}(\Omega)\right)} .
$$

Next we have

$$
\begin{aligned}
\left|\mathscr{E}_{3}\right|= & \mid \gamma_{M}\left(\left(\boldsymbol{w}-P_{\boldsymbol{X}} \boldsymbol{w}, \varrho \mathcal{W}\right)\right)+\gamma_{M}\left(\left(\varrho\left(I-P_{I}\right) P_{\boldsymbol{X}} \boldsymbol{w}, \mathcal{W}-\overline{\mathcal{W}}\right)\right) \\
& \quad-\gamma_{E}\left(\left(\left(I-P_{I}\right) \Delta \boldsymbol{w}, \mathcal{W}-\overline{\mathcal{W}}\right)\right) \mid \\
\leqslant & C\left\|\left(I-P_{\boldsymbol{X}}\right) \boldsymbol{w}\right\|_{L_{1}\left(I ; \boldsymbol{L}_{2}(\Omega)\right)}\left\|\varrho^{1 / 2} \mathcal{W}\right\|_{L_{\infty}\left(I ; \boldsymbol{L}_{2}(\Omega)\right)} \\
& \quad+C\left(\gamma_{M}\left\|\left(I-P_{I}\right) P_{\boldsymbol{X}} \boldsymbol{w}\right\|_{L_{r}(I ; \boldsymbol{X})}+\gamma_{E}\left\|\left(I-P_{I}\right) \Delta \boldsymbol{w}\right\|_{L_{r}(I ; \boldsymbol{X})}\right)\|\mathcal{W}-\overline{\mathcal{W}}\|_{L_{p}\left(I ; \boldsymbol{X}^{\prime}\right)} \\
\leqslant & C\left\|\left(I-P_{\boldsymbol{X}}\right) \boldsymbol{w}\right\|_{L_{1}\left(I ; \boldsymbol{L}_{2}(\Omega)\right)}\left\|\varrho^{1 / 2} \mathcal{W}\right\|_{L_{\infty}\left(I ; \boldsymbol{L}_{2}(\Omega)\right)} \\
& +C k^{3}\left(\gamma_{M}\|\ddot{\boldsymbol{u}}\|_{L_{r}(I ; \boldsymbol{X})}+\gamma_{E}\|\Delta \dddot{\boldsymbol{u}}\|_{L_{r}(I ; \boldsymbol{X})}\right)\left\|\varrho^{1 / 2} \dot{\mathcal{W}}\right\|_{L_{p}\left(I ; \boldsymbol{X}^{\prime}\right)} .
\end{aligned}
$$

Arguing similarly as for $\mathscr{E}_{2}$ we have,

$$
\begin{aligned}
& \left.\left|\mathscr{E}_{4}\right|=\mid a\left(\left(\mathcal{W}-\overline{\mathcal{W}}, \varphi_{0}\left(I-P_{I}\right) P_{\boldsymbol{X}} \boldsymbol{u}\right)\right)\right) \mid \leqslant C k^{3}\left\|\varrho^{1 / 2} \dot{\mathcal{W}}\right\|_{L_{p}\left(I ; \boldsymbol{X}^{\prime}\right)}\|\Delta \ddot{\boldsymbol{u}}\|_{L_{r}(I ; \boldsymbol{X})} \\
& \left|\mathscr{E}_{5}\right| \leqslant \sum_{q=1}^{N_{\varphi}} C k^{3}\left\|\tau_{q}^{1 / 2} \dot{\mathcal{Z}}_{q}\right\|_{L_{\infty}\left(I ; \boldsymbol{L}_{2}(\Omega)\right)}\|\Delta \ddot{\boldsymbol{w}}\|_{L_{1}\left(I ; \boldsymbol{L}_{2}(\Omega)\right)}
\end{aligned}
$$

and also,

$$
\begin{aligned}
\left|\mathscr{E}_{10}+\mathscr{E}_{11}\right| \leqslant C k^{3}( & \left.\sum_{q=1}^{N_{\varphi}}\left\|\tau_{q}^{1 / 2} \dot{\mathcal{Z}}_{q}\right\|_{L_{\infty}(I ; \boldsymbol{X})}^{2}\right)^{1 / 2}\left(\sum_{q=1}^{N_{\varphi}}\left\|\ddot{\boldsymbol{z}}_{q}\right\|_{L_{1}(I ; \boldsymbol{X})}^{2}\right)^{1 / 2} \\
& +C k^{3}\left\|\varrho^{1 / 2} \dot{\mathcal{W}}\right\|_{L_{p}\left(I ; \boldsymbol{X}^{\prime}\right)}\left(\sum_{q=1}^{N_{\varphi}}\left\|\Delta \ddot{\boldsymbol{z}}_{q}\right\|_{L_{r}(I ; \boldsymbol{X})}^{2}\right)^{1 / 2}
\end{aligned}
$$

Using now (29) and the Cauchy-Schwarz inequality for sums we get

$$
\left|\mathscr{E}_{6}+\mathscr{E}_{7}\right| \leqslant 2\left(\sum_{n=1}^{N}\left\|\varrho^{1 / 2}\left(I-P_{\boldsymbol{X}}\right) \dot{\boldsymbol{u}}_{n}^{-}\right\|_{0}^{2}\right)^{1 / 2}\left(\left\|\varrho^{1 / 2} \mathcal{W}_{N}^{+}\right\|_{0}^{2}+\sum_{n=1}^{N}\left\|\varrho^{1 / 2} \llbracket \mathcal{W} \rrbracket_{n}\right\|_{0}^{2}\right)^{1 / 2}
$$

while, again from (29), we have $\mathscr{E}_{8}=\mathscr{E}_{9}=\mathscr{E}_{12}=\mathscr{E}_{13}=0$. Putting all of these together 
and taking $\mathfrak{g}=\mathbf{0}$ gives,

$$
\begin{aligned}
& \left(\varrho \mathcal{W}_{N}^{+},\left(\boldsymbol{W}-\Pi_{w} \boldsymbol{w}\right)_{N}^{-}\right)+a\left(\varphi_{0} \mathcal{U}_{N}^{+},\left(\boldsymbol{U}-\Pi_{u} \boldsymbol{u}\right)_{N}^{-}\right) \\
& \leqslant C\left(\left\|\left(I-P_{\boldsymbol{X}}\right) \dot{\boldsymbol{u}}\right\|_{L_{r}(I ; \boldsymbol{X})}^{2}+\left\|\left(I-P_{\boldsymbol{X}}\right) \dot{\boldsymbol{u}}\right\|_{L_{1}\left(I ; \boldsymbol{L}_{2}(\Omega)\right)}^{2}+\sum_{n=1}^{N}\left\|\left(I-P_{\boldsymbol{X}}\right) \dot{\boldsymbol{u}}_{n}^{-}\right\|_{0}^{2}\right. \\
& +k^{6}\|\Delta \dddot{\boldsymbol{u}}\|_{L_{1}\left(I ; \boldsymbol{L}_{2}(\Omega)\right)}^{2}+k^{6}\|\dddot{\boldsymbol{u}}\|_{L_{r}(I ; \boldsymbol{X})}^{2}+k^{6}\|\Delta \ddot{\boldsymbol{u}}\|_{W_{r}^{1}(I ; \boldsymbol{X})}^{N_{\varphi}} \\
& \left.\quad+k^{6} \sum_{q=1}^{N_{\varphi}}\left\|\ddot{\boldsymbol{z}}_{q}\right\|_{L_{1}(I ; \boldsymbol{X})}^{2}+k^{6} \sum_{q=1}^{1 / 2}\left\|\Delta \ddot{\boldsymbol{z}}_{q}\right\|_{L_{r}(I ; \boldsymbol{X})}^{2}\right)^{N} \\
& \quad \times\left(\left\|\varrho^{1 / 2} \dot{\mathcal{W}}\right\|_{L_{p}\left(I ; \boldsymbol{X}^{\prime}\right)}^{2}+\left\|\varphi_{0}^{1 / 2} \dot{\mathcal{U}}\right\|_{L_{\infty}\left(I ; \boldsymbol{L}_{2}(\Omega)\right)}^{2}+\left\|\varrho^{1 / 2} \mathcal{W}\right\|_{L_{\infty}\left(I ; \boldsymbol{L}_{2}(\Omega)\right)}^{2}\right. \\
& \left.\quad+\sum_{q=1}^{N_{\varphi}}\left\|\tau_{q}^{1 / 2} \dot{\mathcal{Z}}_{q}\right\|_{L_{\infty}(I ; \boldsymbol{X})}^{2}+\left\|\varrho^{1 / 2} \mathcal{W}_{N}^{+}\right\|_{0}^{2}+\sum_{n=1}^{N}\left\|\varrho^{1 / 2} \llbracket \mathcal{W} \rrbracket_{n}\right\|_{0}^{2}\right)^{1 / 2}
\end{aligned}
$$

and we can obtain $\left\|\left(I-P_{\boldsymbol{X}}\right) \dot{\boldsymbol{u}}\right\|_{L_{r}(I ; \boldsymbol{X})} \leqslant C h\|\dot{\boldsymbol{u}}\|_{L_{r}\left(I ; \boldsymbol{H}^{2}(\Omega)\right)}$, for $r \in[1, \infty]$, for the spatial errors using standard arguments.

Now, from (8) we have $\boldsymbol{z}_{q}=(\psi * \dot{\boldsymbol{u}})$ for $\psi(t)=\left(\varphi_{q} / \tau_{q}\right)^{1 / 2} \exp \left(-t / \tau_{q}\right)$ and so using Hölder's inequality for convolutions, $\left\|\boldsymbol{z}_{q}\right\|_{L_{r}(I ; \cdot)} \leqslant \beta_{q}\|\dot{\boldsymbol{u}}\|_{L_{r}(I ; \cdot)}$ because $\|\psi\|_{L_{1}(I)} \leqslant \beta_{q}$. From (9) we then obtain first that $\left\|\dot{\boldsymbol{z}}_{q}\right\|_{L_{r}(I ; \cdot)} \leqslant 2\left(\varphi_{q} / \tau_{q}\right)^{1 / 2}\|\dot{\boldsymbol{u}}\|_{L_{r}(I ; \cdot)}$ and then secondly that $\left\|\ddot{z}_{q}\right\|_{L_{r}(I ; \cdot)} \leqslant C\|\dot{\boldsymbol{u}}\|_{W_{r}^{1}(I ; \cdot)}$.

Using Theorem 4.4 and then choosing $\mathcal{W}_{N}^{+}=\left(\boldsymbol{W}-\Pi_{w} \boldsymbol{w}\right)_{N}^{-}$and $\mathcal{U}_{N}^{+}=\left(\boldsymbol{U}-\Pi_{u} \boldsymbol{u}\right)_{N}^{-}$ gives

$$
\begin{aligned}
\left\|\varrho^{1 / 2}\left(\boldsymbol{W}-\Pi_{w} \boldsymbol{w}\right)_{N}^{-}\right\|_{0}+ & \left\|\varphi_{0}^{1 / 2}\left(\boldsymbol{U}-\Pi_{u} \boldsymbol{u}\right)_{N}^{-}\right\|_{\boldsymbol{X}} \\
& \leqslant C T^{1 / 2+1 / p}\left(1+c_{T}\right) \mathscr{R}(\boldsymbol{u})\left(h+k^{3}+k^{-1 / 2} h^{2}\right) .
\end{aligned}
$$

The proof is then completed by using the triangle inequality and more approximation error bounds for $\left\|\varrho^{1 / 2}\left(\boldsymbol{w}-\Pi_{w} \boldsymbol{w}\right)_{N}^{-}\right\|_{0}+\left\|\varphi_{0}^{1 / 2}\left(\boldsymbol{u}-\Pi_{u} \boldsymbol{u}\right)_{N}^{-}\right\|_{\boldsymbol{X}}$.

roror

The kinetic plus energy error is estimated by terms of order $O\left(h+k^{3}+k^{-1 / 2} h^{2}\right)$ in Theorem 4.5 which, because $h \leqslant c_{T} k$, is of size $O\left(h+k^{3}\right)$ and since we can allow $k \sim h^{q}$ for $q \in(0,1]$ (as $h \rightarrow 0$, because $h=h^{1-q} h^{q} \leqslant h^{q} \sim k$ ), we may have errors of size $O\left(h+h^{3 q}\right)=O\left(h^{\gamma}\right)$ for $\gamma<1$. We illustrate and discuss this later at the end of Section 5 ,

The $O\left(k^{3}\right)$ superconvergence in time in the bound $O\left(h+k^{3}+k^{-1 / 2} h^{2}\right)$ is expected for temporally piecewise linear approximations and was reported in [1, Thm. 2.3, Rem. 2.6] for parabolic problems. The $O(h)$ term is very standard and arises from error bounds for the elliptic projection. The $O\left(k^{-1 / 2} h^{2}\right)$ is more unusual in that it is not seen in error bounds for finite-difference-in-time methods. It arises here because the term $\mathscr{E}_{6}$ in the proof of Theorem 4.5 contains a sum of squared $\boldsymbol{L}_{2}(\Omega)$ spatial errors over all $N$ time levels with no compensating weight of the time step $k$ to kill the growth. Hence the sum of $N \sim k^{-1}$ terms of size $O\left(h^{4}\right)$ is controlled by a bound of order $O\left(k^{-1 / 2} h^{2}\right)$.

The bound in Theorem 4.5 is only optimal if we regard the left hand norms as inseparable. Otherwise, experience tells us that we could expect $\left\|\varrho^{1 / 2}(\boldsymbol{w}-\boldsymbol{W})_{N}^{-}\right\|_{0}=$ 
$O\left(h^{2}+k^{3}+k^{-1 / 2} h^{2}\right)$ and $\left\|\varphi_{0}^{1 / 2}(\boldsymbol{u}-\boldsymbol{U})_{N}^{-}\right\|_{\boldsymbol{X}}=O\left(h+k^{3}+k^{-1 / 2} h^{2}\right)$ - although the first of these these is not proven here.

Furthermore, we can expect that using piecewise polynomials of degree $p>1$ in space would (regularity permitting) result in a bound of size $O\left(h^{p}+k^{3}+k^{-1 / 2} h^{p+1}\right)$ in Theorem 4.5. We can also see that while higher degree temporal DG polynomial approximation would improve the $O\left(k^{3}\right)$ term, it would not affect the factor of $k^{-1 / 2}$.

\section{$5 \quad$ Implementation and results}

The implementation given below is restricted to piecewise linears in time in order to illustrate Theorem 4.5. Unlike Li and Wiberg's method in [17, we do not need an iterative solution but instead eliminate the displacements so the linear block-solve is for just the velocities. Only the main steps are outlined. The formulation includes the case where a traction is imposed on $\Gamma_{N}$ but, to remain consistent with Theorem 4.5 we revert to $\Gamma_{N}=\varnothing$ for the numerical results.

On a given time interval, $I_{n}$, we choose a piecewise linear temporal basis $\theta_{1}, \theta_{2}: I_{n} \rightarrow \mathbb{R}$ and, in (20), write $\boldsymbol{U}(t)=\boldsymbol{U}_{1} \theta_{1}(t)+\boldsymbol{U}_{2} \theta_{2}(t), \boldsymbol{W}(t)=\boldsymbol{W}_{1} \theta_{1}(t)+\boldsymbol{W}_{2} \theta_{2}(t)$ and, for each $q, \boldsymbol{Z}_{q}(t)=\boldsymbol{Z}_{q, 1} \theta_{1}(t)+\boldsymbol{Z}_{q, 2} \theta_{2}(t)$ where $\boldsymbol{U}_{j}, \boldsymbol{W}_{j}, \boldsymbol{Z}_{q, j} \in \boldsymbol{X}^{h}$ for each $q$ and for $j=1,2$. Then, defining,

$$
\mathbf{M}=\int_{t_{n-1}}^{t_{n}}\left(\begin{array}{cc}
\theta_{1}(t) \theta_{1}(t) & \theta_{2}(t) \theta_{1}(t) \\
\theta_{1}(t) \theta_{2}(t) & \theta_{2}(t) \theta_{2}(t)
\end{array}\right) d t
$$

and

$$
A=\int_{t_{n-1}}^{t_{n}}\left(\begin{array}{cc}
\dot{\theta}_{1}(t) \theta_{1}(t) & \dot{\theta}_{2}(t) \theta_{1}(t) \\
\dot{\theta}_{1}(t) \theta_{2}(t) & \dot{\theta}_{2}(t) \theta_{2}(t)
\end{array}\right) d t+\left.\left(\begin{array}{cc}
\theta_{1} \theta_{1} & \theta_{2} \theta_{1} \\
\theta_{1} \theta_{2} & \theta_{2} \theta_{2}
\end{array}\right)\right|_{t_{n-1}}
$$

we can choose $\boldsymbol{\vartheta}=\theta_{i}(t) \boldsymbol{v}$ in (20) and extract the discrete momentum equation,

$$
\begin{gathered}
\mathrm{A}\left(\begin{array}{c}
\left(\varrho \boldsymbol{W}_{1}, \boldsymbol{v}\right) \\
\left(\varrho \boldsymbol{W}_{2}, \boldsymbol{v}\right)
\end{array}\right)+\mathrm{M}\left(\begin{array}{c}
a\left(\varphi_{0} \boldsymbol{U}_{1}, \boldsymbol{v}\right) \\
a\left(\varphi_{0} \boldsymbol{U}_{2}, \boldsymbol{v}\right)
\end{array}\right)+\mathrm{M}\left(\begin{array}{c}
b\left(\boldsymbol{W}_{1}, \boldsymbol{v}\right) \\
b\left(\boldsymbol{W}_{2}, \boldsymbol{v}\right)
\end{array}\right)+\sum_{q=1}^{N_{\varphi}} \mathrm{M}\left(\begin{array}{c}
a\left(\beta_{q} \boldsymbol{Z}_{q, 1}, \boldsymbol{v}\right) \\
a\left(\beta_{q} \boldsymbol{Z}_{q, 2}, \boldsymbol{v}\right)
\end{array}\right) \\
=\left(\begin{array}{c}
\theta_{1}\left(t_{n-1}\right) \\
\theta_{2}\left(t_{n-1}\right)
\end{array}\right)\left(\varrho \boldsymbol{W}_{n-1}^{-}, \boldsymbol{v}\right)+\int_{t_{n-1}}^{t_{n}}\left(\begin{array}{c}
\theta_{1}(t) \\
\theta_{2}(t)
\end{array}\right)\left((\boldsymbol{f}(t), \boldsymbol{v})+(\boldsymbol{g}(t), \boldsymbol{v})_{\Gamma_{N}}\right) d t
\end{gathered}
$$

for all $\boldsymbol{v} \in \boldsymbol{X}^{h}$. Next, choosing $\boldsymbol{\zeta}=\theta_{i}(t) \boldsymbol{v}$ in (20) gives the following discrete enforcement of $\dot{\boldsymbol{u}}=\boldsymbol{w}$ as,

$$
\left(\begin{array}{c}
\boldsymbol{U}_{1} \\
\boldsymbol{U}_{2}
\end{array}\right)=\mathrm{A}^{-1} \mathrm{M}\left(\begin{array}{l}
\boldsymbol{W}_{1} \\
\boldsymbol{W}_{2}
\end{array}\right)+\mathrm{A}^{-1}\left(\begin{array}{c}
\theta_{1}\left(t_{n-1}\right) \boldsymbol{U}_{n-1}^{-} \\
\theta_{2}\left(t_{n-1}\right) \boldsymbol{U}_{n-1}^{-}
\end{array}\right)
$$

and, with this, the momentum equations simplify to,

$$
\begin{aligned}
\mathrm{A}\left(\begin{array}{r}
\left(\varrho \boldsymbol{W}_{1}, \boldsymbol{v}\right) \\
\left(\varrho \boldsymbol{W}_{2}, \boldsymbol{v}\right)
\end{array}\right)+\mathrm{MA}^{-1} \mathrm{M}\left(\begin{array}{r}
a\left(\varphi_{0} \boldsymbol{W}_{1}, \boldsymbol{v}\right) \\
a\left(\varphi_{0} \boldsymbol{W}_{2}, \boldsymbol{v}\right)
\end{array}\right)+\mathrm{M}\left(\begin{array}{c}
b\left(\boldsymbol{W}_{1}, \boldsymbol{v}\right) \\
b\left(\boldsymbol{W}_{2}, \boldsymbol{v}\right)
\end{array}\right) \\
\quad+\sum_{q=1}^{N_{\varphi}} \mathrm{M}\left(\begin{array}{c}
a\left(\beta_{q} \boldsymbol{Z}_{q, 1}, \boldsymbol{v}\right) \\
a\left(\beta_{q} \boldsymbol{Z}_{q, 2}, \boldsymbol{v}\right)
\end{array}\right)=\int_{t_{n-1}}^{t_{n}}\left(\begin{array}{c}
\theta_{1}(t) \\
\theta_{2}(t)
\end{array}\right)\left((\boldsymbol{f}(t), \boldsymbol{v})+(\boldsymbol{g}(t), \boldsymbol{v})_{\Gamma_{N}}\right) d t \\
\quad+\left(\begin{array}{c}
\theta_{1}\left(t_{n-1}\right) \\
\theta_{2}\left(t_{n-1}\right)
\end{array}\right)\left(\varrho \boldsymbol{W}_{n-1}^{-}, \boldsymbol{v}\right)-\mathrm{MA}^{-1}\left(\begin{array}{c}
\theta_{1}\left(t_{n-1}\right) \\
\theta_{2}\left(t_{n-1}\right)
\end{array}\right) a\left(\varphi_{0} \boldsymbol{U}_{n-1}^{-}, \boldsymbol{v}\right) .
\end{aligned}
$$


In a similar way, by choosing $\boldsymbol{\xi}_{q}=\boldsymbol{v} \theta_{i}(t)$ in (20) we obtain,

$$
\left(\tau_{q} \mathrm{~A}+\mathrm{M}\right)\left(\begin{array}{c}
\boldsymbol{Z}_{q, 1} \\
\boldsymbol{Z}_{q, 2}
\end{array}\right)=\beta_{q} \mathrm{M}\left(\begin{array}{l}
\boldsymbol{W}_{1} \\
\boldsymbol{W}_{2}
\end{array}\right)+\tau_{q} \boldsymbol{Z}_{q, n-1}^{-}\left(\begin{array}{c}
\theta_{1}\left(t_{n-1}\right) \\
\theta_{2}\left(t_{n-1}\right)
\end{array}\right)
$$

which can be substituted into the momentum equations to result in a two-by-two block system for $\boldsymbol{W}_{1}$ and $\boldsymbol{W}_{2}$.

To make progress we choose the specific forms $\theta_{1}(t)=1$ and $\theta_{2}(t)=\left(t_{n}-t\right) / k$ and then obtain easily that $A=\frac{1}{2}\left(\begin{array}{ll}2 & 0 \\ 2 & 1\end{array}\right)$ and $M=\frac{k}{6}\left(\begin{array}{ll}6 & 3 \\ 3 & 2\end{array}\right)$. Moreover $A^{-1}=\left(\begin{array}{rr}1 & 0 \\ -2 & 2\end{array}\right)$ and $\mathrm{M}^{-1}=\frac{1}{k}\left(\begin{array}{rr}4 & -6 \\ -6 & 12\end{array}\right)$ and, further, $\mathrm{M}^{-1} \mathrm{~A}=\frac{1}{k}\left(\begin{array}{rr}-2 & -3 \\ 6 & 6\end{array}\right), \mathrm{MA}^{-1}=\frac{k}{6}\left(\begin{array}{rr}0 & 6 \\ -1 & 4\end{array}\right), \mathrm{A}^{-1} \mathrm{M}=\frac{k}{6}\left(\begin{array}{rr}6 & 3 \\ -6 & -2\end{array}\right)$ and $\mathrm{MA}^{-1} \mathrm{M}=\frac{k^{2}}{36}\left(\begin{array}{cc}18 & 12 \\ 6 & 5\end{array}\right)$. After a significant amount of routine calculation we arrive at a specific form of the momentum equations as,

$$
\begin{aligned}
& k^{2}\left[\left(\begin{array}{rr}
3 \varphi_{0}+6 \gamma_{E} k^{-1} & 2 \varphi_{0}+3 \gamma_{E} k^{-1} \\
\varphi_{0}+3 \gamma_{E} k^{-1} & 5 \varphi_{0} / 6+2 \gamma_{E} k^{-1}
\end{array}\right)+\sum_{q=1}^{N_{\varphi}} d_{q} \beta_{q}^{2}\left(\begin{array}{rr}
6\left(3 \tau_{q}+k\right) & 3\left(4 \tau_{q}+k\right) \\
3\left(2 \tau_{q}+k\right) & \left(5 \tau_{q}+2 k\right)
\end{array}\right)\right] \\
& \times\left(\begin{array}{c}
a\left(\boldsymbol{W}_{1}, \boldsymbol{v}\right) \\
a\left(\boldsymbol{W}_{2}, \boldsymbol{v}\right)
\end{array}\right)+\left(\begin{array}{cc}
6+6 \gamma_{M} k & 0+3 \gamma_{M} k \\
6+3 \gamma_{M} k & 3+2 \gamma_{M} k
\end{array}\right)\left(\begin{array}{c}
\left(\varrho \boldsymbol{W}_{1}, \boldsymbol{v}\right) \\
\left(\varrho \boldsymbol{W}_{2}, \boldsymbol{v}\right)
\end{array}\right) \\
& =\int_{t_{n-1}}^{t_{n}}\left(\begin{array}{l}
6 \\
6\left(t_{n}-t\right) / k
\end{array}\right)\left((\boldsymbol{f}(t), \boldsymbol{v})+(\boldsymbol{g}(t), \boldsymbol{v})_{\Gamma_{N}}\right) d t+\left(\begin{array}{l}
6 \\
6
\end{array}\right)\left(\varrho \boldsymbol{W}_{n-1}^{-}, \boldsymbol{v}\right) \\
& -k\left(\begin{array}{l}
6 \\
3
\end{array}\right) a\left(\varphi_{0} \boldsymbol{U}_{n-1}^{-}, \boldsymbol{v}\right)-\sum_{q=1}^{N_{\varphi}} 6 k d_{q} \beta_{q} \tau_{q}\left(\begin{array}{c}
6 \tau_{q}+k \\
3 \tau_{q}+k
\end{array}\right) a\left(\boldsymbol{Z}_{q, n-1}^{-}, \boldsymbol{v}\right) .
\end{aligned}
$$

Once $\boldsymbol{W}_{1}$ and $\boldsymbol{W}_{2}$ are obtained from this we update $\boldsymbol{U}_{1}$ and $\boldsymbol{U}_{2}$ with

$$
\boldsymbol{U}_{1}=k \boldsymbol{W}_{1}+\frac{k}{2} \boldsymbol{W}_{2}+\boldsymbol{U}_{n-1}^{-} \quad \text { and } \quad \boldsymbol{U}_{2}=-k \boldsymbol{W}_{1}-\frac{k}{3} \boldsymbol{W}_{2}
$$

and then obtain $\boldsymbol{Z}_{q, 1}$ and $\boldsymbol{Z}_{q, 2}$ from

$$
\left(\begin{array}{l}
\boldsymbol{Z}_{q, 1} \\
\boldsymbol{Z}_{q, 2}
\end{array}\right)=k d_{q} \beta_{q}\left(\begin{array}{rr}
6 \tau_{q}+k & 3 \tau_{q} \\
-6 \tau_{q} & k-2 \tau_{q}
\end{array}\right)\left(\begin{array}{l}
\boldsymbol{W}_{1} \\
\boldsymbol{W}_{2}
\end{array}\right)+2 d_{q} \tau_{q} \boldsymbol{Z}_{q, n-1}^{-}\left(\begin{array}{l}
3 \tau_{q}-k \\
3 k
\end{array}\right) .
$$

We now give the results of some computations designed specifically to illustrate the convergence rates of the algorithm derived above.

To verify that the observed convergence rates agree with those stated in Theorem 4.5 we manufacture an exact solution and choose the data consistent with that solution. For this we take $\Omega:=(0,1)^{2}$, the unit square, with $T=12 \pi$ and we consider an exact solution in the form,

$$
\boldsymbol{u}=\overline{\boldsymbol{u}}(\boldsymbol{x}) \mathcal{T}(t) \quad \text { for } \quad \overline{\boldsymbol{u}}(\boldsymbol{x}):=\left(\begin{array}{l}
u_{1} \\
u_{2}
\end{array}\right)=16\left(x^{2}-x\right)\left(y^{2}-y\right)\left(\begin{array}{l}
1 \\
1
\end{array}\right)
$$

where $\mathcal{T}(t)=t+B \cos (t)$ for a constant $B$ (taken as $B=0$ or $B=1$ below). Then $\boldsymbol{w}=\overline{\boldsymbol{u}} \mathcal{T}^{\prime}(t)$ and we see that $\boldsymbol{u}$ satisfies the requirements of Theorem4.5. As mentioned earlier, we consider the material to be isotropic, homogeneous and synchronous and then, on using (2) with (4) and the assumption of Rayleigh damping as in (13) we can obtain the loads once the coefficients are defined. For these we take $\varrho=1, \lambda=1$ and 


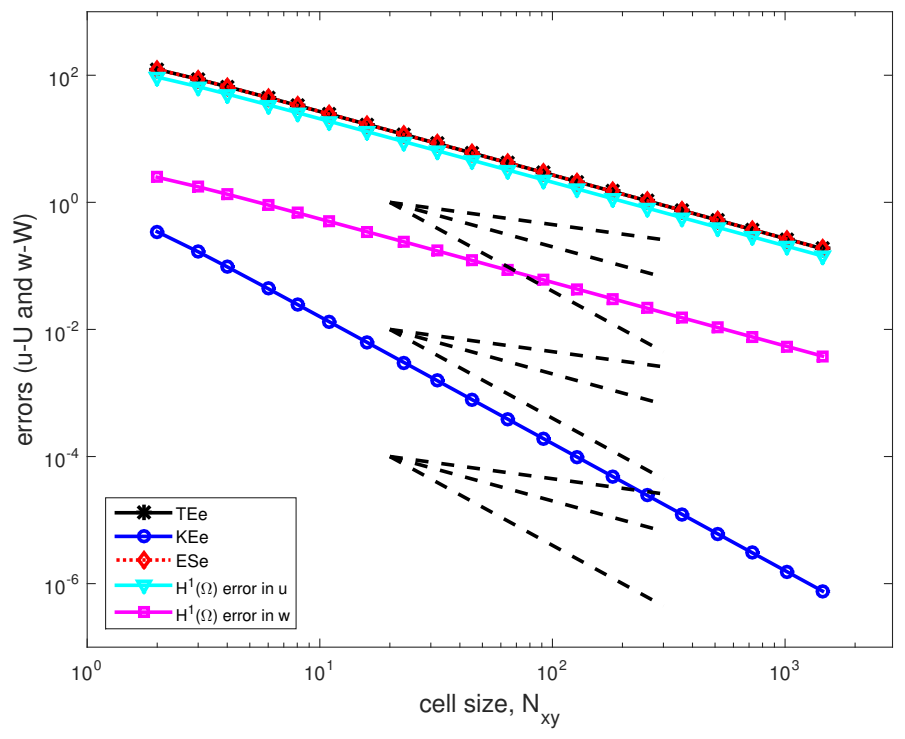

Figure 1: Errors for Example I, the dashed lines indicate rates of $N_{x y}^{-p}$ for $p=1 / 2,1,2$. In this example $B=0$ and there are no viscoelastic terms - the Galerkin errors are due only to the spatial approximation.

$\mu=1$, with Rayleigh damping given by $\gamma_{M}=2, \gamma_{E}=1$, and three-term, $N_{\varphi}=2$, viscoelasticity given by $\left(\varphi_{1}, \tau_{1}\right)=(0.35,0.1)$ and $\left(\varphi_{2}, \tau_{2}\right)=(0.15,0.05)$ for all but the first example below. In the discrete scheme we used an $N_{x y} \times N_{x y}$ mesh of isosceles triangles with piecewise linear elements and a uniform time step of $k=T / N_{t}$, for $N_{t} \in \mathbb{N}$. We set $h=N_{x y}^{-1}$

In Examples I, II, III and IV below the errors, ' $\boldsymbol{e}$ ', are reported in the kinetic energy norm, KEe, $\left\|\varrho^{1 / 2} \boldsymbol{e}_{w}(T)\right\|_{0}$ for $\boldsymbol{e}_{w}(T):=\boldsymbol{w}(T)-\boldsymbol{W}_{N}^{-}$, the elastic strain energy norm, ESe, $\left\|\varphi_{0}^{1 / 2} \boldsymbol{e}_{u}(T)\right\|_{\boldsymbol{X}}$ for $\boldsymbol{e}_{u}(T):=\boldsymbol{u}(T)-\boldsymbol{U}_{N}^{-}$, the total energy norm, TEe, $\left(\left\|\varrho^{1 / 2} \boldsymbol{e}_{w}(T)\right\|_{0}^{2}+\left\|\varphi_{0}^{1 / 2} \boldsymbol{e}_{u}(T)\right\|_{\boldsymbol{X}}^{2}\right)^{1 / 2}$, as well as the $\boldsymbol{H}^{1}(\Omega)$ norm for both $\boldsymbol{e}_{u}(T)$ and $\boldsymbol{e}_{w}(T)$.

These results were computed using a $65 \mathrm{~Gb}$ Intel Xeon E5-2640 v4 CPU (2.40GHz). We used the 2017.1.0 FEniCS (see Logg et al. in [19] and fenicsproject.org) docker image started with

docker run -ti ... quay.io/fenicsproject/stable:2017.1.0

(... indicates that superfluous details are omitted) on 20 December 2018. A custom image built for this paper can be pulled in docker with

docker pull variationalform/fem:dgcgwave,

(see https://hub.docker.com/r/variationalform/fem) and then run with docker run -ti variationalform/fem:dgcgwave.

The command cd fenics followed by ./bigrun.sh -J 3 | tee runmeout.txt will, for a suite of twelve test cases, produce the error results up to $N_{x y}=\operatorname{int}\left(2^{3 / 2}\right)$ in the results directory. Examples 5,10,11 and 12 (resp.) of those correspond to examples I, II, III and IV (resp.) below. Use $-\mathrm{J} 7$ to go up to $N_{x y}=\operatorname{int}\left(2^{7 / 2}\right)$ and so on (but it will take longer).

In Example I we set $B=0$ and $N_{t}=4$ and switch the viscoelasticity off by setting 


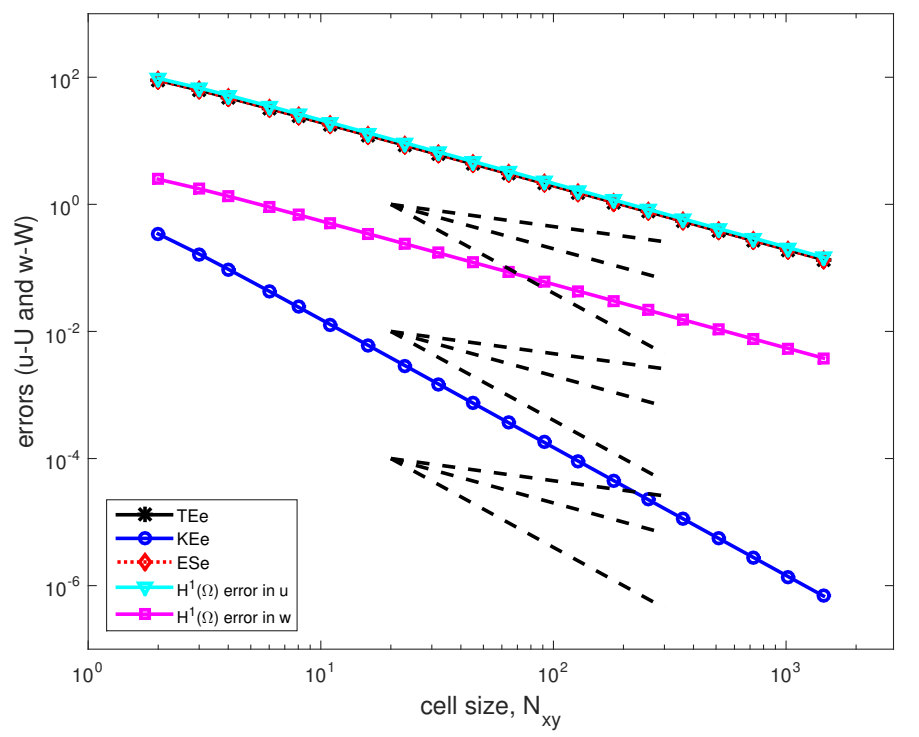

Figure 2: Errors for Example II, the dashed lines indicate rates of $N_{x y}^{-p}$ for $p=1 / 2,1,2$ and $k \sim h^{2 / 3}$.

$\varphi_{0}=1$, the Galerkin errors are therefore due only to the spatial approximation and are shown in Figure 1. In each of Examples II, III and IV we choose $B=1$ with the coefficients given earlier. For these examples we choose the time step $k \sim h^{q}$, specifically

$$
k=\frac{T}{\max \left\{1, \operatorname{int}\left(\frac{T}{h^{q}}\right)\right\}},
$$

for $q=2 / 3,1 / 3$ and $1 / 6$, and show the results in Figure 2 for Example II, Figure 3 for Example III and Figure 4 for Example IV. In each case $h \sim h^{1-q} k \leqslant c_{T} k$ and so the conditions of Theorem 4.5 are satisfied. Indeed, the order of convergence as predicted by the Theorem becomes $h+k^{3}+k^{-1 / 2} h^{2}=h+h^{3 q}+h^{2-q / 2}$ which is $O(h)$ for $q=2 / 3$ and $q=1 / 3$, but $O\left(h^{1 / 2}\right)$ for $q=1 / 6$. In Figures 1 and 2 we can see clearly that the spatial error in the $\boldsymbol{H}^{1-p}(\Omega)$ norm is $O\left(h^{1+p}\right)$ for $p=0$ and $p=1$. This is expected (although the $p=1$ case is not proven here) but we also see from Figure 3 that when $q=1 / 3$ the $O(h)$ term stems from the $k^{3}=h^{3 q}$ part of the estimate and so the expected $O\left(h^{2}\right)$ error associated with the kinetic energy error (the $\boldsymbol{L}_{2}(\Omega)$ error in $\dot{\boldsymbol{u}})$ does not appear. Furthermore, the $O\left(h^{1 / 2}\right)$ error for $q=1 / 6$ is beginning to asymptotically show in Figure 4 for all except the dominant elastic strain energy error in $\boldsymbol{u}$. The curves for these $\boldsymbol{H}^{1}(\Omega)$ type errors in displacement appear indistinguishable in each of Figures 1, 2 and 3. This indicates that those errors are dominated by the $O(h)$ spatial error component for these values of $N_{x y}$ and not by the $O\left(h^{3 q}\right)$ associated with the $O\left(k^{3}\right)$ term.

\section{Conclusions}

We have extended the formulation and a priori error analysis given in [12] from the acoustic wave equation to a viscodynamic system incorporating Rayleigh damping. The elastic term in the Rayleigh damping introduces a multiplicative $T^{1 / 2}$ growth in the constant but otherwise the error bound is consistent with that obtained in [12], 


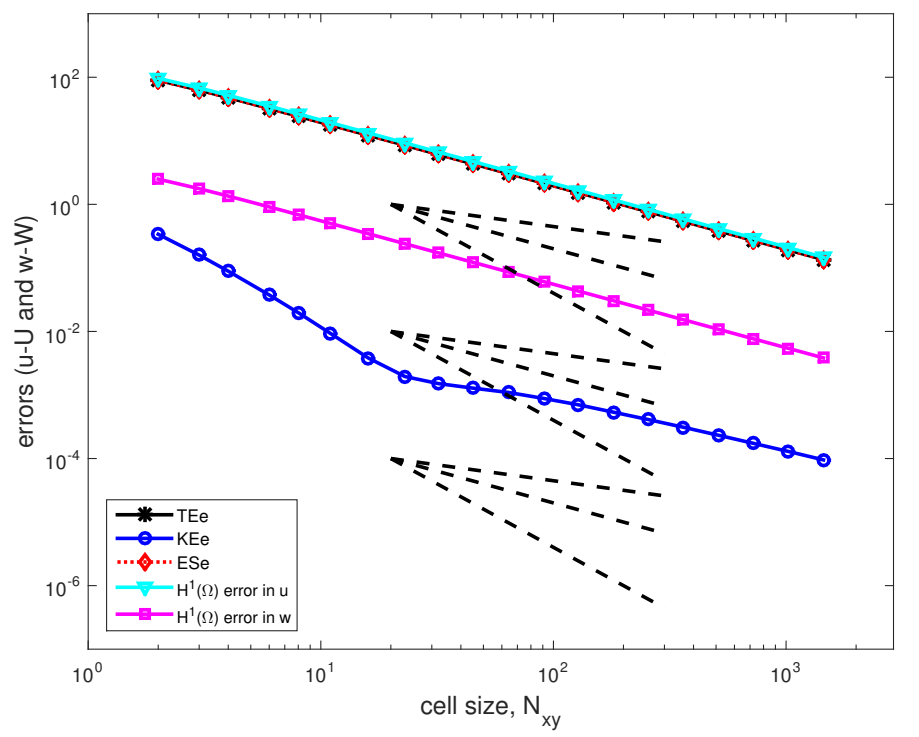

Figure 3: Errors for Example III, the dashed lines indicate rates of $N_{x y}^{-p}$ for $p=1 / 2,1,2$ and $k \sim h^{1 / 3}$.

with a constant that grows a priori with $T^{1 / 2}$ and also with the norms in $\mathscr{R}(u)$ (which could of course be simplified at the expense of introducing more powers of $T$ ). However, Gronwall's inequality is not used and so we can expect that this bound is of high enough quality to afford confidence in long-time integration.

The results of some numerical experiments are given in Figure1 for Example I, Figure 2 for Example II, Figure 3 for Example III and Figure 4 for Example IV and these demonstrate that the a priori estimate given in Theorem 4.5 is optimal. They also demonstrate that the $\boldsymbol{L}_{2}(\Omega)$ kinetic energy errors alone can converge at a rate faster than that predicted by the theorem.

\section{References}

[1] Kenneth Eriksson and Claes Johnson. Adaptive finite element methods for parabolic problems. I: a linear model problem. SIAM J. Numer. Anal., 28:43-77, 1991.

[2] J. D. Ferry. Viscoelastic properties of polymers. John Wiley and Sons Inc., 1970.

[3] Donald A. French. A space-time finite element method for the wave equation. Comput. Methods Appl. Mech. Engrg., 107:145-157, 1993.

[4] Donald A. French and Todd E. Peterson. A continuous space-time finite element method for the wave equation. Math. Comput., 65:491-506, 1996.

[5] J. M. Golden and G. A. C. Graham. Boundary value problems in linear viscoelasticity. Springer-Verlag, 1988.

[6] Yunqing Huang, Jichun Li, and Wei Yang. Solving metamaterial Maxwell's equations via a vector wave integro-differential equation. Comput. Math. Appl., 63:1597-1606, 2012. 


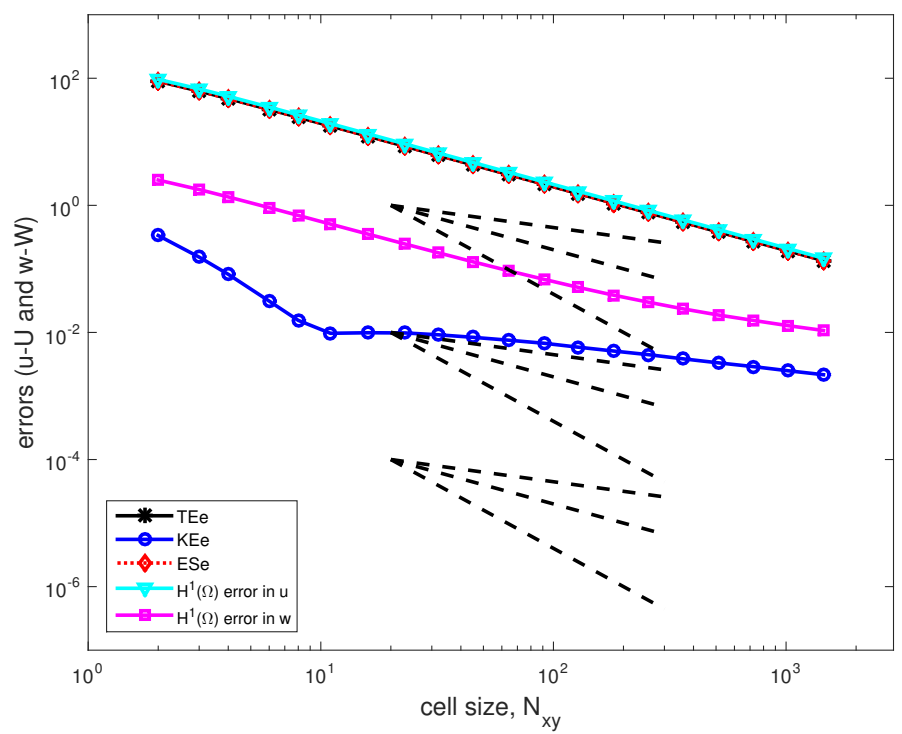

Figure 4: Errors for Example IV, the dashed lines indicate rates of $N_{x y}^{-p}$ for $p=1 / 2,1,2$ and $k \sim h^{1 / 6}$.

[7] Thomas J. R. Hughes and Gregory M. Hulbert. Space-time finite element methods for elastodynamics: formulations and error estimates. Comp. Meth. Appl. Mech. Eng., 66:339-363, 1988.

[8] Gregory M. Hulbert. Time finite element method for structural dynamics. Int. J. Numer. Meth. Engng., 33:307-331, 1992.

[9] Gregory M. Hulbert and Thomas J. R. Hughes. Space-time finite element methods for second-order hyperbolic equations. Comp. Meth. Appl. Mech. Eng., 84:327348, 1990.

[10] A. R. Johnson. Modeling viscoelastic materials using internal variables. The Shock and Vibration Digest, 31:91-100, 1999.

[11] A. R. Johnson, A. Tessler, and M. Dambach. Dynamics of thick viscoelastic beams. Journal of Engineering Materials and Technology, 119:273 - 278, 1997.

[12] Claes Johnson. Discontinuous Galerkin finite element methods for second order hyperbolic problems. Comp. Meth. Appl. Mech. Eng., 107:117-129, 1993.

[13] Carola Kruse and Simon Shaw. Time-decoupled high order continuous space-time finite element schemes for the heat equation. SIAM J. Sci. Comput., 36:B1-B13, 2014. doi: 10.1137/130914589; BURA: http://bura.brunel.ac.uk/handle/ 2438/8120; (BICOM Tech. Rep. 13/7, see www.brunel.ac.uk/bicom).

[14] Carola Kruse, Matthias Maischak, Simon Shaw, John R Whiteman, Stephen E Greenwald, Malcolm J Birch, Mark P Brewin, H T Banks, Zackary R Kenz, and Shuhua Hu. High order space-time finite element schemes for acoustic and viscodynamic wave equations with temporal decoupling. Int. J. Numer. Meth. Engng., 98:131-156, 2014. doi: 10.1002/nme.4631; BURA: TBC; (BICOM Tech. Rep. 13/6, see ww. brunel.ac.uk/bicom). 
[15] Jichun Li. Numerical convergence and physical fidelity analysis for Maxwell's equations in metamaterials. Comput. Methods Appl. Mech. Engrg., 198:3161$3172,2009$.

[16] Jichun Li and Yitung Chen. Analysis of a time-domain finite element method for 3-D Maxwell's equations in dispersive media. Comput. Methods Appl. Mech. Engrg., 195:4220-4229, 2006.

[17] X. D. Li and N.-E. Wiberg. Implementation and adaptivity of a space-time finite element method for structural dynamics. Comput. Methods Appl. Mech. Engrg., 156:211-229, 1998 .

[18] F. J. Lockett. Nonlinear viscoelastic solids. Academic Press, 1972.

[19] Anders Logg, Kent-Andre Mardal, Garth N. Wells, et al. Automated Solution of Differential Equations by the Finite Element Method. Springer, 2012.

[20] William McLean. Fast summation by interval clustering for an evolution equation with memory. Siam J. Sci. Comput, 34:A3039-A3056, 2012.

[21] J. Nečas and I. Hlaváček. Mathematical theory of elastic and elastico-plastic bodies: an introduction. Studies in Applied Mathematics 3. Elsevier, 1981.

[22] Thomas Richter, Andreas Springer, and Boris Vexler. Efficient numerical realization of discontinuous Galerkin methods for temporal discretization of parabolic problems. Numer. Math., 124:151-182, 2013.

[23] Béatrice Rivière, Simon Shaw, and J.R. Whiteman. Discontinuous Galerkin finite element methods for dynamic linear solid viscoelasticity problems. Numer. Methods Partial Differential Equations, 23:1149-1166, 2007. See also report 05/7 at www. brunel.ac.uk/bicom.

[24] Achim Schädle, María López-Fernández, and Christian Lubich. Fast and oblivious convolution quadrature. SIAM J. Sci. Comput., 28:421-438, 2006.

[25] Simon Shaw. Finite element approximation of Maxwell's equations with Debye memory. Advances in Numerical Analysis, doi:10.1155/2010/923832, 2010, 2010. Technical Report 10/2 at www. brunel.ac.uk/bicom.

[26] I. H. Sloan and V. Thomée. Time discretization of an integro-differential equation of parabolic type. SIAM J. Numer. Anal., 23:1052-1061, 1986.

[27] Vidar Thomée. Galerkin finite element methods for parabolic problems. Number 25 in Springer series in computational mathematics. Springer-Verlag, Berlin Heidelberg, 1997.

[28] T. Werder, K. Gerdes, D. Schötzau, and C. Schwab. hp-discontinuous Galerkin time stepping for parabolic problems. Comput. Methods Appl. Mech. Engrg., 190:6685-6708, 2001.

[29] Xiaoming Zhou, Xiaoning Liu, and Gengkai Hu. Elastic metamaterials with local resonances: an overview. Theoretical and Applied Mechanics Letters, 2:041001-2 - 041001-12, 2012. 